\title{
Varieties $\boldsymbol{n}$-covered by curves of degree $\delta$
}

\author{
Luc Pirio and Francesco Russo
}

\begin{abstract}
We continue the study, began by classical geometers and considered more recently in [33] and [52], of complex projective varieties $X \subset \mathbb{P}^{N}$ such that through $n \geq 2$ general points of $X$ there passes an irreducible curve of degree $\delta$.
\end{abstract}

Mathematics Subject Classification (2010). 14M22, $17 \mathrm{C} 36$.

Keywords. Projective variety, rationally connected variety, Jordan algebra.

\section{Introduction}

The theory of rationally connected varieties is quite recent and was formalized in [10], [36] and [35], although these varieties were intensively studied from different points of view by classical algebraic geometers, see for example [19], [59], [56], [6], [5], [42].

An important result in this theory, see Theorem IV.3.9 in [35], asserts that through $n$ general points of a smooth rationally connected complex variety $X$ there passes an irreducible rational curve, which can be taken also to be smooth as soon as $\operatorname{dim}(X) \geq 3$. From this one deduces that for $\operatorname{dim}(X) \geq 3$ a fixed smooth curve of arbitrary genus can be embedded into $X$ in such a way that it passes through $n$ arbitrary fixed general points. When a (rationally connected) variety $X$ is embedded in some projective space $\mathbb{P}^{N}$ (or more generally when a polarization or an arbitrary Cartier divisor is fixed on $X$ ), one can consider the property of being generically $n$-(rationally) connected by (rational) curves of a fixed degree $\delta$.

This stronger condition depends on the embedding, on the number $n \geq 2$, on the degree $\delta \geq 1$ and natural constraints for the existence of such varieties immediately appear.

In this paper we shall study complex irreducible projective varieties $X=X(r+$ $1, n, \delta) \subset \mathbb{P}^{N}$ of dimension $\operatorname{dim}(X)=r+1$ such that through $n \geq 2$ general points there passes an irreducible curve $C$ of degree $\delta \geq 1$ or more generally pairs $(X, D)$ with $D$ a Cartier divisor on a proper irreducible complex variety $X$ which is $n$-covered by irreducible curves $C$ such that $(D \cdot C)=\delta \geq n-1$. It is well known that a $X(r+1,2,1) \subset \mathbb{P}^{N}$ is necessarily a $\mathbb{P}^{r+1}$ linearly embedded in $\mathbb{P}^{N}$ 
and that a non-degenerate $X(r+1, n, n-1) \subset \mathbb{P}^{n+r-1}$ is a variety of minimal degree $n-1$. The smooth $X(r+1,2,2)$ 's were recently classified in [33] (see also [48] for a generalization to the polarized case). Without some reasonable restrictions the classification of varieties $X(r+1, n, \delta) \subset \mathbb{P}^{N}$ becomes immediately extremely difficult and out of reach, especially for singular varieties.

Recently (see [63], [51] and also [49]), it has been realized that the study of these varieties is also closely related to an important question in web geometry, namely the algebraization of webs of maximal rank. In order to solve this problem of web geometry, it was proved in [52] that the dimension of the linear span of such varieties satisfies the inequality

$$
\operatorname{dim}(\langle X(r+1, n, \delta)\rangle) \leq \bar{\pi}(r, n, \delta)-1,
$$

see Section 1 . Here $\bar{\pi}(r, n, \delta)=\pi(r, n, d)$ with $d=\delta+r(n-1)+2$ where $\pi(r, n, d)$ stands for the Castelnuovo-Harris bound function for the geometric genus of nondegenerate irreducible $r$-dimensional projective varieties $Y \subset \mathbb{P}^{n+r-1}$ of degree $d$, see [30] and also Section 4.2 for some details and definitions. The bound (0.1) is proved geometrically via the iteration of projections from general osculating spaces to $X(r+1, n, \delta)$ determined by the irreducible curves of degree $\delta$ which $n$-cover the variety. This is a classical tool used also by del Pezzo, Enriques and Castelnuovo to bound the dimension of linear systems on surfaces, see [11], [12], [22], Section 7 in [16] and [52]. The above bound also reveals a connection with Castelnuovo theory of linear systems on curves and with the so-called Castelnuovo varieties, see [30] and [14]. From this point of view, non-degenerate varieties $X=X(r+1, n, \delta) \subset \mathbb{P}^{\bar{\pi}(r, n, \delta)-1}$, denoted from now on by $X=\bar{X}(r+1, n, \delta)$, are the extremal ones and they are subject to very strong restrictions - e.g. they are rational and through $n$ general points there passes a unique rational normal curve of degree $\delta$, see [52] and Theorem 2.2 and Theorem 2.4 below. Due to these numerous geometrical properties, it is possible in many cases to obtain a complete classification, see for example [52] or Theorem 2.2, Theorem 5.7, Corollary 5.9 and Corollary 5.12 below in this paper.

Some basic results of [52] are generalized here in Theorem 2.4. One proves the bound $h^{0}\left(\mathcal{O}_{X}(D)\right) \leq \bar{\pi}(r, n, \delta)$ for a Cartier divisor $D$ on a proper irreducible variety $X$ of dimension $r+1, n$-covered by irreducible curves $C$ such that $(D \cdot C)=\delta \geq n-1$. We also show that equality holds if and only if $\phi_{|D|}$ maps $X$ birationally onto a $\bar{X}(r+1, n, \delta)$. Furthermore, if $h^{0}\left(\mathcal{O}_{X}(D)\right)=\bar{\pi}(r, n, \delta)$ then $X$ is rational and through $n \geq 2$ general points there passes a unique smooth rational curve $C$ such that $(D \cdot C)=\delta$.

Another consequence of the previous bound is that under the same hypothesis we have $D^{r+1} \leq \delta^{r+1} /(n-1)^{r}$ if $D$ is nef, see Theorem 3.1. This is a generalization of a result usually attributed to Fano in the case $n=2$, see for example Proposition V.2.9 of [35].

The classical roots of this type of results go back to C. Segre, [59], who proved that $\operatorname{dim}(\langle X(2,2,2)\rangle) \leq 5$ and that $\bar{X}(2,2,2) \subset \mathbb{P}^{5}$ is projectively equivalent to the 
Veronese surface. Bompiani generalized this result in $[6]$ to $\operatorname{dim}(\langle X(r+1,2, \delta)\rangle) \leq$ $\left(\begin{array}{c}r+1+\delta \\ r+1\end{array}\right)-1$ with equality holding if and only if $\bar{X}(r+1,2, \delta)$ is projectively equivalent to the $\delta$-Veronese embedding of $\mathbb{P}^{r+1}$, see Theorem 2.2 here and also [32] and [64].

A lot of examples of $\bar{X}(r+1, n, \delta)$ (for arbitrary $n \geq 2, r \geq 1$ and $\delta \geq n-1$ ) have been described in [52] via the theory of Castelnuovo varieties and their construction will be briefly recalled in Section 4.2. The main result of [52] ensures that these examples of Castelnuovo type are the only ones except possibly when $n>2, r>1$ and $\delta=2 n-3$.

Here we consider in detail the last open case, that is the classification of varieties $\bar{X}(r+1, n, 2 n-3)$, especially for $n=3$. We immediately point out that there are examples of $\bar{X}(r+1, n, 2 n-3)$ of dimension at least 3 that are not of Castelnuovo type. For $n=3$ these varieties share very special structures being related to the theory of cubic Jordan algebras, see Section 4.3. Indeed interesting examples of $\bar{X}(r+1,3,3) \subset \mathbb{P}^{2 r+3}$ are the so-called twisted cubics over complex Jordan algebras of rank 3, see [46]. There is an infinite family of such varieties: the Segre embeddings $\mathbb{P}^{1} \times Q^{r} \subset \mathbb{P}^{2 r+3}$, where $Q^{r} \subset \mathbb{P}^{r+1}$ is an irreducible hyperquadric, and also four smooth exceptional examples associated to the four simple cubic Jordan algebras (these four varieties are also known as Lagrangian Grassmannians) and other examples constructed by considering cubic Jordan algebras naturally arising from associative algebras, see Section 4.3. For an arbitrary $\bar{X}(r+1,3,3) \subset \mathbb{P}^{2 r+3}$ we consider the birational projection onto $\mathbb{P}^{r+1}$ from a general tangent space. By studying the geometry of this birational map we are able to give an explicit parametrization of these varieties and also to associate to them a quadro-quadric Cremona transformation from the projectivization of a general affine tangent space onto a hyperplane in $\mathbb{P}^{r+1}$, see Theorem 5.2. From this unexpected connection we deduce the classification of arbitrary $\bar{X}(r+1,3,3)$ for $r \leq 3$ (even if our method actually works also for $r=4$ or for bigger values of $r$ ), see Corollary 5.9 and Corollary 5.12. As a consequence we also prove that the base locus of a quadro-quadric Cremona transformation and the base locus of its inverse are projectively equivalent so that essentially these transformations are involutions, Corollary 5.3, a fact which seems to have been overlooked as far as we know. Moreover in Theorem 5.7 we provide the classification of all smooth $\bar{X}(r+1,3,3)$, showing that they are either smooth rational normal scrolls (hence of Castelnuovo type) or the Segre embeddings of $\mathbb{P}^{1} \times Q^{r}$ or one of the four Lagrangian Grassmannians. Our approach yields also a geometrical direct proof of the classification of all cubic Jordan algebras whose associated variety is smooth, showing that they are either simple (Lagrangian Grassmannian) or semi-simple ( $\mathbb{P}^{1} \times Q^{r}$ with $Q^{r}$ smooth).

The paper is organized as follows. In Section 1 we introduce some definitions and explain the notation. We also recall the main steps for the proof of the bound (0.1). In Section 2, a modern version of Bompiani's theorem [6] is proved, Theorem 2.2. Then, inductively via osculating projections and the study of the rational map $\phi_{|D|}$ 
we prove in Theorem 2.4 the bound on the dimension of linear systems of Cartier divisors described above and the consequences of the maximality of this dimension. In Section 3 we deduce from the bound on the dimension of the linear span a general bound for the degree of nef divisors on varieties $n$-covered by irreducible curves. In Section 4, after describing the $\bar{X}(r+1, n, \delta)$ of Castelnuovo type in Section 4.2, we construct new examples of $\bar{X}(r+1, n, \delta)$ when $r>1, n>2$ and $\delta=2 n-3$. In particular we describe in detail examples of $\bar{X}(r+1,3,3)$ of Jordan type in Section 4.3. In Section 4.5 we present some examples of $\bar{X}(3, n, 2 n-3)$ which are not of Castelnuovo type for $n=4,5,6$. Section 5 concerns the classification of several classes of $\bar{X}(r+1,3,3)$ under different assumptions either on $r$, Corollary 5.9 and Corollary 5.12, or on the smoothness of the variety, Theorem 5.7. We also discuss some open problems pointing towards the equivalence of these apparently unrelated objects: varieties $\bar{X}(r+1,3,3)$, quadro-quadric Cremona transformations of $\mathbb{P}^{r}$ and complex Jordan algebras of rank three and of dimension $r+1$.

Acknowledgements. Both authors are grateful to Ciro Ciliberto for some discussions at different stages of the preparation of the paper and for a lot of suggestions leading to an improvement of the exposition. The first author has considered the problem studied here when developing a research on webs in common with Jean-Marie Trépreau. He learned a lot from the numerous discussions with him on this subject during last years. The second author expresses his gratitude to Paltin Ionescu for a direct or indirect but undoubtedly rich intellectual exchange of ideas and points of view on some contents of the paper, for his interest in the results and for a lot of remarks which improved the presentation.

\section{Preliminaries and notation}

We shall consider irreducible varieties $X$ which are projective, or proper, over the complex field $\mathbb{C}$.

We will use the following notation: $r, n$ and $\delta$ are positive integers such that $n-1 \leq \delta$. Then one defines $\rho=\left\lfloor\frac{\delta}{n-1}\right\rfloor$ and $\epsilon=\delta-\rho(n-1) \in\{0, \ldots, n-2\}$. One also defines $m=\epsilon+1>0$ and $m^{\prime}=n-1-m \geq 0$ so that $m+m^{\prime}=n-1$. Finally, for any integer $k$, one sets $k^{+}=\max \{0, k\}$.

For classification results there is no restriction in supposing that an irreducible variety $X \subset \mathbb{P}^{N}$ is non-degenerate. Otherwise $\langle X\rangle \subset \mathbb{P}^{N}$ will denote the linear span of $X$ in $\mathbb{P}^{N}$, that is the smallest linear subspace of $\mathbb{P}^{N}$ containing $X$. For computational reasons, when dealing with classification results, we shall define $r$ such that $\operatorname{dim}(X)=r+1$.

Let $x$ be a smooth point of $X$. For any $\ell \in \mathbb{N}$, we denote by $\operatorname{Osc}_{X}^{\ell}(x) \subset \mathbb{P}^{N}$ the $\ell$-th order osculation space of $X$ at $x$. If $\psi:\left(\mathbb{C}^{r+1}, 0\right) \rightarrow(X, x), u \mapsto \psi(u)$ is a regular local parametrization of $X$ at $x$, then $\operatorname{Osc}_{X}^{\ell}(x)$ can be defined as the 
projective subspace $\left\langle\partial^{|\alpha|} \psi(0) / \partial u^{\alpha}\left|\alpha \in \mathbb{N}^{r+1},\right| \alpha \mid \leq \ell\right\rangle \subset \mathbb{P}^{N}$. This space can also be defined more abstractly as the linear subspace spanned by the $\ell$-th order infinitesimal neighborhood of $X$ at $x$ and also generalized to the case of arbitrary Cartier divisors $D$ on $X$. Indeed, for every integer $\ell \in \mathbb{N}$, let $\mathcal{P}_{X}^{\ell}(D)$ denote the $\ell$-th principal part bundle (or $\ell$-th jet bundle) of $\mathcal{O}_{X}(D)$. For every linear subspace $V \subseteq H^{0}\left(X, \mathcal{O}_{X}(D)\right)$ we have a natural homomorphism of sheaves

$$
\phi^{\ell}: V \otimes \mathcal{O}_{X} \rightarrow \mathcal{P}_{X}^{\ell}(D),
$$

sending a section $s \in V$ to its $\ell$-th jet $\phi_{x}^{\ell}(s)$ evaluated at $x \in X$, that is $\phi_{x}^{\ell}(s)$ is represented in local coordinates by the Taylor expansion of $s$ at $x$, truncated after the order $\ell$. Taking a smooth point $x \in X \subset \mathbb{P}^{N}=\mathbb{P}(V)$ (Grothendieck's notation) and $\mathcal{O}_{X}(D)=\mathcal{O}_{X}(1)$, it is easily verified that $\operatorname{Osc}_{X}^{\ell}(x)=\mathbb{P}\left(\operatorname{Im}\left(\phi_{x}^{\ell}\right)\right)$. If $x \in X$ is a smooth point, the previous definitions yield $\operatorname{dim}\left(\operatorname{Osc}_{X}^{\ell}(x)\right) \leq \operatorname{rk}\left(\mathscr{P}_{X}^{\ell}\right)-1=$ $\left(\begin{array}{c}r+1+\ell \\ r+1\end{array}\right)-1$ and in general it is expected that equality holds at general points of $X \subset \mathbb{P}^{N}$ as soon as $N \geq\left(\begin{array}{c}r+1+\ell \\ r+1\end{array}\right)-1$. In this case, we shall say that the osculation of order $\ell$ of $X$ at $x$ is regular.

A curve $C \subset \mathbb{P}^{N}$ is a rational normal curve of degree $\delta$ if it is rational, smooth, of degree $\delta$ and its linear span in $\mathbb{P}^{N}$ has dimension $\delta$. In other terms: the restriction of $\left|\mathcal{O}_{\mathbb{P}^{N}}(1)\right|$ to $C$ is the complete linear system of degree $\delta$ on $C \simeq \mathbb{P}^{1}$.

Let us define the following Castelnuovo-Harris function which bounds the geometric genus of irreducible projective varieties, see Theorem 4.2 below:

$$
\pi(r, n, d)=\sum_{\sigma \geq 0}\left(\begin{array}{c}
\sigma+r-1 \\
\sigma
\end{array}\right)(d-(\sigma+r)(n-1)-1)^{+} .
$$

We will also use the following function that is closely related to $\pi(r, n, d)$ :

$$
\bar{\pi}(r, n, \delta):=m\left(\begin{array}{c}
r+\rho+1 \\
r+1
\end{array}\right)+m^{\prime}\left(\begin{array}{l}
r+\rho \\
r+1
\end{array}\right),
$$

for $\delta \geq n-1$ fixed with $\rho=\left\lfloor\frac{\delta}{n-1}\right\rfloor, m=\delta-\rho(n-1)+1$ and $m^{\prime}=n-1-m$.

It is not difficult to prove that $\bar{\pi}(r, n, \delta)=\pi(r, n, d)$, see [52] for details.

An irreducible projective variety $X \subset \mathbb{P}^{N}$ of dimension $r+1$ that is $n$-covered by a family of irreducible curves of degree $\delta$ will be denoted by $X(r+1, n, \delta) \subset \mathbb{P}^{N}$. In most of the cases we shall also assume that $X(r+1, n, \delta) \subset \mathbb{P}^{N}$ is non-degenerate.

For reader's convenience we reproduce here some basic results of [52] on varieties $X=X(r+1, n, \delta) \subset \mathbb{P}^{N}$. For an irreducible curve $C \subset \mathbb{P}^{N}$ of degree $\delta$, for nonnegative integers $a_{1}, \ldots, a_{\kappa}$, with $\kappa>0$ fixed and such that $\sum_{i=1}^{\kappa}\left(a_{i}+1\right) \geq \delta+1$ and for $x_{1}, \ldots, x_{\kappa} \in C$ pairwise distinct smooth points, one has:

$$
\langle C\rangle=\left\langle\operatorname{Osc}_{C}^{a_{i}}\left(x_{i}\right) \mid i=1, \ldots, \kappa\right\rangle
$$


(otherwise there would exist a hyperplane $H \subsetneq\langle C\rangle$ containing $\left\langle\operatorname{Osc}_{C}^{a_{i}}\left(x_{i}\right)\right| i=$ $1, \ldots, \kappa\rangle$ and $\delta=\operatorname{deg}(C)=\operatorname{deg}(H \cap C) \geq \sum_{i=1}^{\kappa}\left(a_{i}+1\right)$, contrary to our assumption).

Let $X=X(r+1, n, \delta) \subset \mathbb{P}^{N}$ and let $\Sigma$ be a fixed $n$-covering family of irreducible curves of degree $\delta$ on $X$. If $x_{1}, \ldots, x_{n-1}$ are distinct general points on $X$ one can consider the subfamily $\Sigma_{x_{1}, \ldots, x_{n-1}}=\left\{C \in \Sigma \mid x_{i} \in C\right.$ for $\left.i=1, \ldots, n-1\right\}$. Since $\Sigma$ is $n$-covering, the family $\Sigma_{x_{1}, \ldots, x_{n-1}}$ covers $X$ and we can also assume that the general curve in this family is non-singular at $x_{1}, \ldots, x_{n-1}$. Let $\left\{a_{1}, \ldots, a_{n-1}\right\}$ be a set of $n-1$ non-negative integers such that $\sum_{i=1}^{n-1}\left(a_{i}+1\right) \geq \delta+1$. By (1.4) and since $\operatorname{Osc}_{C}^{a_{i}}\left(x_{i}\right) \subset \operatorname{Osc}_{X}^{a_{i}}\left(x_{i}\right)$ for every $i=1, \ldots, n-1$, it comes that $\langle C\rangle \subseteq\left\langle\operatorname{Osc}_{X}^{a_{i}}\left(x_{i}\right) \mid i=1, \ldots, n-1\right\rangle$ for a general $C \in \Sigma_{x_{1}, \ldots, x_{n-1}}$. Since the elements of $\Sigma_{x_{1}, \ldots, x_{n-1}}$ cover $X$, one obtains

$$
\langle X\rangle=\left\langle\operatorname{Osc}_{X}^{a_{i}}\left(x_{i}\right) \mid i=1, \ldots, n-1\right\rangle .
$$

Therefore for these varieties we deduce that $\operatorname{dim}(\langle X\rangle)+1 \leq \sum_{i=1}^{n-1}\left(\begin{array}{c}r+1+a_{i} \\ r+1\end{array}\right)$. Taking $a_{1}=\cdots=a_{m}=\rho$ and $a_{m+1}=\cdots=a_{n-1}=\rho-1$ and recalling (1.3), we obtain the following result for arbitrary $X=X(r+1, n, \delta)$, see [52]:

$$
\operatorname{dim}(\langle X\rangle) \leq \bar{\pi}(r, n, \delta)-1 .
$$

Recall that for $\bar{\pi}(r, n, \delta)$ defined in (1.3) we have $\bar{\pi}(r, n, \delta)=\pi(r, n, d)$, where the Castelnuovo-Harris bound $\pi(r, n, d)$ is defined in (1.2) and where $d$ is defined as a function of $\delta$ by $d=\delta+r(n-1)+2$. Since $\operatorname{dim}\left(\operatorname{Osc}_{X}^{\ell}(x)\right) \leq\left(\begin{array}{c}r+1+\ell \\ r+1\end{array}\right)-1$ for any point $x \in X$ and for any integer $\ell \in \mathbb{N}$, we deduce an immediate consequence of (1.5) that for a non-degenerate $X=X(r+1, n, \delta) \subset \mathbb{P}^{\bar{\pi}(r, n, \delta)-1}$ the following hold:

(i) the osculation of order $\rho$ of $X$ at a general point $x \in X$ is regular, that is

$$
\operatorname{dim}\left(\operatorname{Osc}_{X}^{\rho}(x)\right)=\left(\begin{array}{c}
r+1+\rho \\
r+1
\end{array}\right)-1
$$

(ii) if $x_{1}, \ldots, x_{n-1}$ are general points of $X$, then

$$
\langle X\rangle=\left(\bigoplus_{i=1}^{m} \operatorname{Osc}_{X}^{\rho}\left(x_{i}\right)\right) \oplus\left(\bigoplus_{j=1}^{m^{\prime}} \operatorname{Osc}_{X}^{\rho-1}\left(x_{m+j}\right)\right)=\mathbb{P}^{\bar{\pi}(r, n, \delta)-1} .
$$

From now on an irreducible non-degenerate projective variety $X=X(r+$ $1, n, \delta) \subset \mathbb{P}^{\bar{\pi}(r, n, \delta)-1}$ will be denoted by $\bar{X}(r+1, n, \delta) \subset \mathbb{P}^{\bar{\pi}(r, n, \delta)-1}$ or simply by $\bar{X}(r+1, n, \delta)$. In the next sections we shall describe the notable geometric properties of these varieties and of their covering families. 


\section{Rationality of $\bar{X}(r+1, n, \delta)$ and of the general curve of the $n$-covering family}

The following simple remark, which is surely well known to the experts, will play a central role several times in our analysis, see also Lemma 2.2 in [32] and [52] for related statements. Since we were unable to find a precise reference for the generality needed, we also include a proof.

Lemma 2.1. Let $\phi: X \rightarrow X^{\prime}$ be a dominant rational map between proper varieties of the same dimension, let $\Sigma$ be an irreducible n-covering family of irreducible curves on $X$ and let $\Sigma^{\prime}$ be the induced $n$-covering family on $X^{\prime}$. If $X^{\prime}$ is projective, if the restriction of $\phi$ to a general curve $C \in \Sigma$ induces a morphism birational onto its image and if through $n$-general points of $X^{\prime}$ there passes a unique curve $C^{\prime} \in \Sigma^{\prime}$, then the same is true for $\Sigma$ on $X$ and moreover $\phi$ is a birational map.

Proof. There exists a desingularization $\alpha: \tilde{X} \rightarrow X$ with $\tilde{X}$ projective and a morphism $\tilde{\phi}: \tilde{X} \rightarrow X^{\prime}$ solving the indeterminacies of $\phi$. Thus without loss of generality we can assume $X$ smooth and projective, that $\phi$ is a morphism and that $\phi$ restricted to a general $C \in \Sigma$ is a morphism birational onto its image. The morphism $\phi$ is generically étale by generic smoothness, i.e. there exists an open set $U^{\prime} \subseteq X^{\prime}$ such that letting $U=\phi^{-1}\left(U^{\prime}\right)$, then $\phi_{\mid U}: U \rightarrow U^{\prime}$ is an étale morphism. Let $d=\operatorname{deg}(\phi)=\operatorname{deg}\left(\phi_{\mid U}\right) \geq 1$. We shall prove that $d=1$.

Fix $x_{1}, \ldots, x_{n-1} \in U$ general points. There exists an open subset $U_{1} \subseteq U$ such that for every $x \in U_{1}$, there passes exactly $s \geq 1$ curves in $\Sigma$ through $x_{1}, \ldots, x_{n-1}$ and $x$. Since $\phi$ is a proper morphism we can also take $U_{1}=\phi^{-1}\left(U_{1}^{\prime}\right)$ with $U_{1}^{\prime} \subseteq U^{\prime}$ open. Let $x^{\prime} \in U_{1}^{\prime}$ be a (general) point and let $\phi^{-1}\left(x^{\prime}\right)=\left\{\tilde{x}_{1}, \ldots, \tilde{x}_{d}\right\}$. Let $x_{l}^{\prime}=\phi\left(x_{l}\right), l=1, \ldots, n-1$, and let $\widetilde{C}_{i, 1}, \ldots, \widetilde{C}_{i, s}$ be the curves of $\Sigma$ passing through $x_{1}, \ldots, x_{n-1}, \tilde{x}_{i}$. Then for every $j=1, \ldots, s$, the curves $\phi\left(\widetilde{C}_{i, j}\right)$ belong to $\Sigma^{\prime}$ and they pass through $x_{1}^{\prime}, \ldots, x_{n-1}^{\prime}, x^{\prime}$ so that they coincide with the unique curve $C^{\prime} \in \Sigma^{\prime}$ having this property. Since $\phi$ is a local isomorphism near $\tilde{x}_{i}$, we deduce $s=1$. In particular through $n$ general points of $X$ there passes a unique curve belonging to $\Sigma$.

Then $\tilde{x}_{i} \in \widetilde{C}_{i}, x_{1} \in \widetilde{C}_{i}$ and $\phi\left(\widetilde{C}_{i}\right)=C^{\prime}$ for every $i=1, \ldots, d$. Since $\phi$ is also a local isomorphism at $x_{1}$ since $x_{1} \in U$, we see that $\widetilde{C}_{1}=\cdots=\widetilde{C}_{d}=C$. Since $C \in \Sigma$ is general, by hypothesis $\phi_{\mid C}: C \rightarrow C^{\prime}$ is a morphism birational onto its image, yielding $d=1$ because $\tilde{x}_{i} \in C, \phi\left(\tilde{x}_{i}\right)=x^{\prime}$ for every $i=1, \ldots, d$, and by the generality of $x^{\prime}$ we can also suppose that $\phi^{-1}\left(x^{\prime}\right)$ consists only of a point.

We begin to study general properties of $\bar{X}(r+1, n, \delta)$ 's starting from the case $n=2$. This case was classically considered by Bompiani in [6], where the proof was essentially provided for surfaces. Under the assumption that the general 2covering curve is smooth and rational, this result was also obtained by Ionescu in [32], Theorem 2.8. A similar but stronger result holding in the analytic category 
and not only for complex algebraic varieties has been proved recently by Trépreau in $[64]^{1}$.

Theorem 2.2. An irreducible projective variety $X=\bar{X}(r+1,2, \delta) \subset \mathbb{P}^{\left(\begin{array}{c}r+1+\delta \\ r+1\end{array}\right)-1}$ is projectively equivalent to the Veronese manifold $v_{\delta}\left(\mathbb{P}^{r+1}\right)$. In particular every curve in the 2-covering family is a rational normal curve of degree $\delta$ in the given embedding and there exists a unique such curve passing through two distinct points of $X$.

Proof. By definition $\rho=\delta$ so that by (1.7), for $x \in X$ general we have

$$
\left.\operatorname{dim}\left(\operatorname{Osc}_{X}^{\delta-1}(x)\right)=\left(\begin{array}{l}
r+\delta \\
r+1
\end{array}\right)-1 \quad \text { and } \quad \operatorname{Osc}_{X}^{\delta}(x)=\mathbb{P}^{\left({ }^{r+1+\delta}+\delta\right.}\right)-1
$$

Now let $x \in X$ be a fixed general point and let $T=\operatorname{Osc}_{X}^{\delta-1}(x)=\mathbb{P}^{\left(\begin{array}{c}r+\delta \\ r+1\end{array}\right)-1}$. Let $p_{T}: X \rightarrow \mathbb{P}^{\bar{\pi}(r-1,2, \delta)-1}$ be the restriction to $X$ of the projection from $T$. The rational map $p_{T}$ is given by the linear system $\left|D_{x}\right|$ cut on $X$ by hyperplanes containing $T$ so that the corresponding hyperplane sections have a point of multiplicity $\delta$ at $x \in X$. A general irreducible curve of degree $\delta$ passing through $x$ is thus contracted by $p_{T}$. Let $X_{T}=\overline{p_{T}(X)}$. We have $\left\langle X_{T}\right\rangle=\mathbb{P}^{\bar{\pi}(r-1,2, \delta)-1}$ since $\langle X\rangle=\mathbb{P}^{\bar{\pi}(r, 2, \delta)-1}$.

We claim that $X_{T}$ is projectively equivalent to $\nu_{\delta}\left(\mathbb{P}^{r}\right) \subset \mathbb{P}^{\bar{\pi}(r-1,2, \delta)-1}$. Indeed, let $\pi: \mathrm{Bl}_{x}(X) \rightarrow X$ be the blow-up of $X$ at $x$, let $E=\mathbb{P}^{r}$ be the exceptional divisor and let $p_{T}^{\prime}=p_{T} \circ \pi: \mathrm{Bl}_{x}(X) \rightarrow X_{T}$ be the induced rational map. The restriction of $p_{T}^{\prime}$ to $E$ is a rational dominant map from $\mathbb{P}^{r}$ to $X_{T} \subset \mathbb{P}^{\bar{\pi}(r-1,2, \delta)-1}$ given by a sublinear system of $\left|\mathcal{O}_{\mathbb{P}} r(\delta)\right|$ of dimension $\bar{\pi}(r+1,2, \delta)-1$ so that it is given by the

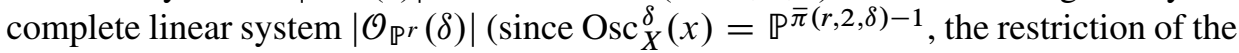
strict transform of the linear system of hyperplane sections containing $\operatorname{Osc}_{X}^{\delta-1}(x)$ to $E$ is not zero). Thus the restriction of $p_{T}^{\prime}$ to $E$ induces an isomorphism between $E$ and $X_{T}$ given by $\left|\mathcal{O}_{\mathbb{P}} r(\delta)\right|$, proving the claim. Moreover since a general curve $C \in \Sigma$ is not contracted by $p_{T}$, we have that $p_{T}(C)$ is a curve on $X_{T}$ of degree $\delta^{\prime} \leq \delta$. Thus $p_{T}(C)$ is a smooth rational curve of degree $\delta, T \cap C=\emptyset$, the rational map $p_{T}$ is defined along $C$ and it gives an isomorphism between $C$ and $p_{T}(C)$.

By solving the indeterminacies of $p_{T}^{\prime}$, we can suppose that there exists a smooth variety $\tilde{X}$, a birational morphism $\phi: \tilde{X} \rightarrow X$ and a morphism $\tilde{p}_{T}: \tilde{X} \rightarrow X_{T} \simeq$ $v_{\delta}\left(\mathbb{P}^{r}\right)$ such that $p_{T} \circ \phi=\tilde{p}_{T}$. Let $\phi^{*}\left(\left|D_{x}\right|\right)=F_{x}+\left|\widetilde{D}_{x}\right|$ with $\left|\widetilde{D}_{x}\right|$ base point free and let $\left|\bar{D}_{x}\right|=\tilde{p}_{T}^{*}\left(\left|\mathcal{O}_{\mathbb{P}} r(1)\right|\right)$. Then $\widetilde{D}_{x} \sim \delta \bar{D}_{x}$ and $\operatorname{dim}\left(\left|\bar{D}_{x}\right|\right) \geq r$. Moreover for the strict transform of a general curve $C_{x}$ in $\Sigma$ passing through $x$ we have $\left(\widetilde{D}_{x} \cdot C_{x}\right)=$ 0 and $\left(F_{x} \cdot C_{x}\right)=\delta$ while for the strict transform of a general curve $C \in \Sigma$ we have $\left(\widetilde{D}_{x} \cdot C\right)=\delta$. Thus $\left(\bar{D}_{x} \cdot C_{x}\right)=0$ and $\left(\bar{D}_{x} \cdot C\right)=1$.

\footnotetext{
${ }^{1}$ Trépreau's version of Bompiani's theorem is the following: let $(X, x) \subset \mathbb{P}^{N}$ be a smooth germ of a $(r+1)$-dimensional analytic variety with regular $\delta$-th order osculation $($ at $x)$. Assume that $X$ is 1 -covered by (germs of) rational curves of degree $\delta$ passing through $x$ that are (generically) smooth at this point. Then $X \subset v_{\delta}\left(\mathbb{P}^{r+1}\right)$.
} 
Letting $T^{\prime}=\operatorname{Osc}_{X}^{\delta-1}\left(x^{\prime}\right)$ with $x^{\prime} \in X$ general and performing the same analysis we can also suppose that on $\tilde{X}$ the rational map $p_{T^{\prime}} \circ \phi=\tilde{p}_{T^{\prime}}$ is defined and that there exists a linear system $\left|\bar{D}_{x^{\prime}}\right|$ such that $\operatorname{dim}\left(\left|\bar{D}_{x^{\prime}}\right|\right) \geq r,\left(\bar{D}_{x^{\prime}} \cdot C_{x^{\prime}}\right)=0$ for general $C_{x^{\prime}} \in \Sigma_{x^{\prime}}$ and $\left(\bar{D}_{x^{\prime}} \cdot C\right)=1$ for general $C \in \Sigma$. Since a general $C_{x^{\prime}}$ in $\Sigma_{x^{\prime}}$ does not pass through $x$ we have $\left|\bar{D}_{x}\right| \neq\left|\bar{D}_{x^{\prime}}\right|$. On the other hand for $x \in X$ general, the linear systems $\left|\bar{D}_{x}\right|$ vary in the same linear system $|D|$ on $\tilde{X}$ since $\tilde{X}$ is rationally connected.

Thus $\operatorname{dim}(|D|) \geq r+1,(D \cdot C)=1$ for the strict transform of a general curve $C$ in $\Sigma$ and $C$ does not intersect the base locus of $|D|$ by the previous analysis. Let $s+1=\operatorname{dim}(|D|)$ and let $\psi=\psi_{|D|}: \tilde{X} \rightarrow \tilde{X}^{\prime} \subseteq \mathbb{P}^{s+1}$ be the associated rational map. Since $\psi(C)$ is a line passing through two general points of $X^{\prime}$, we deduce $X^{\prime}=\mathbb{P}^{s+1}$ and $r=s$. Moreover by Lemma 2.1 the rational map $\psi$ is birational. Hence there exists a birational map $\varphi=\phi \circ \psi^{-1}: \mathbb{P}^{r+1} \rightarrow X$ sending a general line in $\mathbb{P}^{r+1}$ onto a general curve of degree $\delta$ in $\Sigma$. Composing $\varphi$ with the inclusion $X \subset \mathbb{P}^{\bar{\pi}(r, 2, \delta)-1}$ we get a birational map from $\mathbb{P}^{r+1}$ given by a sublinear system of $\left|\mathcal{O}_{\mathbb{P} r+1}(\delta)\right|$ of dimension $\left(\begin{array}{c}r+1+\delta \\ r+1\end{array}\right)-1$, that is $\varphi$ is given by the complete linear system $\left|\mathcal{O}_{\mathbb{P}} r+1(\delta)\right|$. In conclusion $X \subset \mathbb{P}^{\bar{\pi}(r, 2, \delta)-1}$ is projectively equivalent to the Veronese manifold $v_{\delta}\left(\mathbb{P}^{r+1}\right)$.

The rationality and the smoothness of the general member $\Sigma$ of the 2-covering family of a $\bar{X}(r+1,2, \delta)$ can also be deduced differently. Indeed in the previous proof we saw that the linear system of hyperplane sections having a point of multiplicity greater than or equal to $\delta$ at a general $x \in X$ cuts a general $C \in \Sigma_{x}$ in the Cartier divisor $\delta x$. By varying $x$ on $C$ we see that this property holds for the general point of $C$. Thus the smoothness and rationality of a general element of $\Sigma$ are consequences of the following classical and surely well-known result, which seems to go back to Veronese, [65], at least in the projective version. The proof is well known and left to the reader.

Lemma 2.3. Let $C$ be an irreducible projective curve. Then:

(1) if $C \subset \mathbb{P}^{N}$ is non-degenerate and of degree $\delta$, then $N \leq \delta$ and the following conditions are equivalent:

(a) $N=\delta$ and $C \subset \mathbb{P}^{\delta}$ is a rational normal curve of degree $\delta$;

(b) for a general $x \in C$ there exists a hyperplane $H_{x} \subset \mathbb{P}^{N}$ such that $H_{x} \cap X=$ $\delta \cdot x$ as schemes.

(2) The following conditions are equivalent:

(a') $C$ is a smooth rational curve;

$\left(\mathrm{b}^{\prime}\right)$ there exists a Cartier divisor $D$ of degree $\delta \geq 1$ on $C$ such that $\operatorname{dim}(|D|)=\delta$.

$\left(\mathrm{c}^{\prime}\right) \mathcal{O}_{C}\left(\delta \cdot x_{1}\right) \simeq \mathcal{O}_{C}\left(\delta \cdot x_{2}\right)$ for some $\delta \geq 1$ and for $x_{1}, x_{2} \in C$ general points. 
Via Lemma 2.3 one could also prove differently Theorem 2.2 above following the steps of Mori's characterization of projective spaces given in [45] because in this case the family of smooth rational curves $\Sigma_{x}$ is easily seen to be proper.

We now investigate the higher dimensional versions of Lemma 2.3 from the point of view of proper varieties $n$-covered by irreducible curves of degree $\delta$ with respect to some fixed divisor $D$, providing generalizations of Theorem 2.8 in [32] and of Theorem 4.4 in [1]. Part (1) below has been obtained in [52] while (i) is an abstract version of (0.1).

Theorem 2.4. Let $X$ be an irreducible proper variety of dimension $r+1$ and let $D$ be a Cartier divisor on $X$. Suppose that through $n \geq 2$ general points of $X$ there passes an irreducible curve $C$ such that $(D \cdot C)=\delta \geq n-1$. Then:

(i) $h^{0}\left(X, \mathcal{O}_{X}(D)\right) \leq \bar{\pi}(r, n, \delta)$;

(ii) Equality holds in (i) if and only if $\phi_{|D|}$ maps $X$ birationally onto a $\bar{X}(r+$ $1, n, \delta) \subseteq \mathbb{P}^{\bar{\pi}(r, n, \delta)-1}$. In this case the general deformation of $C$ does not intersect the indeterminacy locus of $\phi_{|D|}$.

(iii) If equality holds in (i), then

(a) the variety $X$ is rational;

(b) the general deformation $\bar{C}$ of $C$ is a smooth rational curve and through $n$ general points of $X$ there passes a unique smooth rational curve $\bar{C}$ such that $(D \cdot \bar{C})=\delta$.

In particular:

(1) $a \bar{X}(r+1, n, \delta) \subset \mathbb{P}^{\bar{\pi}(r, n, \delta)-1}$ is rational, the general curve of the $n$-covering family is a rational normal curve of degree $\delta$ and through $n$ general points of $X$ there passes a unique rational normal curve of degree $\delta$;

(2) $a \bar{X}(r+1, n, \delta) \subset \mathbb{P}^{\bar{\pi}(r, n, \delta)-1}$ is a linear birational projection of $v_{\delta}\left(\mathbb{P}^{r+1}\right)$ (or equivalently, a $\bar{X}(r+1, n, \delta)$ is the birational image of $\mathbb{P}^{r+1}$ given by a linear system of hypersurfaces of degree $\delta$ and dimension $\bar{\pi}(r, n, \delta)-1)$.

Proof. Suppose $h^{0}\left(\mathcal{O}_{X}(D)\right) \geq \bar{\pi}(r, n, \delta) \geq 2$ and let

$$
\phi=\phi_{|D|}: X \rightarrow X^{\prime} \subseteq \mathbb{P}\left(H^{0}\left(\mathcal{O}_{X}(D)\right)=\mathbb{P}^{N} .\right.
$$

The variety $X^{\prime} \subseteq \mathbb{P}^{N}$ is irreducible, non-degenerate, of $\operatorname{dimension} \operatorname{dim}\left(X^{\prime}\right)=$ $s+1 \leq r+1$ and is $n$-covered by irreducible curves of degree $\delta^{\prime} \leq \delta$. Therefore by (1.6) one gets

$$
h^{0}\left(\mathcal{O}_{X}(D)\right) \leq \bar{\pi}\left(s, n, \delta^{\prime}\right) \leq \bar{\pi}(r, n, \delta),
$$

yielding $h^{0}\left(\mathcal{O}_{X}(D)\right)=\bar{\pi}(r, n, \delta)$ and $X^{\prime}=\bar{X}(r+1, n, \delta)$. We have thus proved the bound (i) and also that if equality holds then $X^{\prime}=\bar{X}(r+1, n, \delta)$. 
One implication of the first part of (ii) is trivial and we shall prove only the nontrivial implication and the second part of (ii). If $h^{0}\left(\mathcal{O}_{X}(D)\right)=\bar{\pi}(r, n, \delta)$, then by the previous analysis $\operatorname{dim}\left(X^{\prime}\right)=r+1$ and $\operatorname{deg}(\phi(\bar{C}))=\delta$ for a general deformation $\bar{C}$ of $C$.

Now we shall prove the birationality of $\phi$ and part (iii) for $n=2$. Later we shall treat the general case $n>2$. If $n=2$, it follows from Theorem 2.2 that $X^{\prime}=\bar{X}(r+1,2, \delta)$ is projectively isomorphic to $v_{\delta}\left(\mathbb{P}^{r+1}\right)$ so that $\phi(\bar{C})$ is the unique rational normal curve of degree $\delta$ passing through two general points of $X^{\prime}$. Let $|\bar{D}|$ be the linear system on $\bar{C}$ obtained by restricting $|D|$ to $\bar{C}$. Then $\operatorname{deg}(\bar{D})=\delta$ and $\operatorname{dim}(|\bar{D}|)=\delta$ since $\phi(\bar{C})$ is a rational normal curve of degree $\delta$. Part (2) of Lemma 2.3 implies that $\bar{C}$ is a smooth rational curve, that $\phi$ is defined along $\bar{C}$ and that the restriction of $\phi$ to $\bar{C}$ is an isomorphism onto its image. Thus from Lemma 2.1 we deduce that $\phi$ is birational and that through 2 general points of $X$ there passes a unique smooth rational curve $\bar{C}$ such that $(D \cdot \bar{C})=\delta$.

Suppose $n>2$ and recall the following notation, see Section 1: $\rho=\lfloor\delta /(n-1)\rfloor$, $m=\delta-\rho(n-1)+1$ and $m^{\prime}=n-1-m$. Let $x_{1}, \ldots, x_{n-1}$ be $n-1$ general points on $X^{\prime}=\bar{X}(r+1, n, \delta) \subset \mathbb{P}^{\bar{\pi}(r, n, \delta)-1}$. By (1.7) and (1.8), we know that the osculating spaces $\operatorname{Osc}_{X^{\prime}}^{\rho}\left(x_{i}\right)$ and $\operatorname{Osc}_{X^{\prime}}^{\rho-1}\left(x_{m+j}\right)$ (for $i=1, \ldots, m$ and $j=1, \ldots, m^{\prime}$ ) have the maximal possible dimension and are in direct sum in the ambient space, yielding a decomposition

$$
\mathbb{P}^{\bar{\pi}(r, n, \delta)-1}=\left\langle X^{\prime}\right\rangle=\operatorname{Osc}_{X^{\prime}}^{\rho}\left(x_{1}\right) \oplus S
$$

where

$$
S=\left(\bigoplus_{i=2}^{m} \operatorname{Osc}_{X^{\prime}}^{\rho}\left(x_{i}\right)\right) \oplus\left(\bigoplus_{j=1}^{m^{\prime}} \operatorname{Osc}_{X^{\prime}}^{\rho-1}\left(x_{m+j}\right)\right)
$$

Let

$$
p_{S}: X^{\prime} \rightarrow L=\operatorname{Osc}_{X^{\prime}}^{\rho}\left(x_{1}\right)=\mathbb{P}^{\bar{\pi}(r, 2, \rho)-1}
$$

be the restriction to $X^{\prime}$ of the linear projection from $S$ onto $L$ and let $X_{S}^{\prime}=\overline{p_{S}\left(X^{\prime}\right)}$. Let $\Sigma_{x_{2}, \ldots, x_{n-1}}$ be the family consisting of curves in the covering family $\Sigma$ passing through $x_{2}, \ldots, x_{n-1}$. The family $\Sigma_{x_{2}, \ldots, x_{n-1}}$ is 2 -covering and a general $C \in$ $\Sigma_{x_{2}, \ldots, x_{n-1}}$ has contact of order at least $(m-1)(\rho+1)+m^{\prime} \rho$ with $S$ and is not contracted by $p_{S}$. Thus the irreducible curve $C_{S}=p_{S}(C)$ has degree $\rho^{\prime} \leq \delta-$ $(m-1)(\rho+1)-m^{\prime} \rho=\rho$. The projections of the curves in $\Sigma_{x_{2}, \ldots, x_{n-1}}$ produce a 2-covering family of irreducible curves of degree $\rho^{\prime}$ on the non-degenerate irreducible variety $X_{S}^{\prime} \subset \mathbb{P}^{\bar{\pi}(r, 2, \rho)-1}$. Then (1.6) implies that $X_{S}^{\prime}=\bar{X}(r+1,2, \rho)$ is projectively equivalent to $v_{\rho}\left(\mathbb{P}^{r+1}\right)$ so that $C_{S} \subset X_{S}^{\prime}$ is a rational normal curve of degree $\rho$ by Theorem 2.2. Moreover, $C$ is a rational normal curve of degree $\delta$ by part (2) of Lemma 2.3 since the restriction of $p_{S}$ to $C$ is given by a linear system of degree $\rho$ and $p_{S}(C)=C_{S}$ is a rational normal curve of degree $\rho$.

The dominant rational map $p_{S}$ is birational and through $n \geq 2$ points of $X^{\prime}$ there passes a unique rational normal curve of degree $\delta$ by Lemma 2.1. Thus (1) is proved 
for every $n \geq 2$. Applying once again Lemma 2.1 to $\phi: X \rightarrow X^{\prime}$ we immediately deduce also for $n>2$ that the map $\phi$ is birational and that through $n>2$ points of $X$ there passes a unique smooth rational curve $\bar{C}$ such that $(D \cdot \bar{C})=\delta$, proving (a) and (b) of (iii).

To prove (2), let $\varphi=p_{S}^{-1}: \mathbb{P}^{r+1} \rightarrow X^{\prime}=\bar{X}(r+1, n, \delta)$. The birational map $\varphi$ sends a general line $l \subset \mathbb{P}^{r+1}$ onto a general element $C \in \Sigma_{x_{2}, \ldots, x_{n-1}}$. The composition of $\varphi$ with the natural inclusion $X^{\prime}=\bar{X}(r+1, n, \delta) \subset \mathbb{P}^{\bar{\pi}}(r, n, \delta)-1$ is given by a sublinear system of $\left|\mathcal{O}_{\mathbb{P} r+1}(\delta)\right|$ of dimension $\bar{\pi}(r, n, \delta)-1$, showing that $X^{\prime}=\bar{X}(r+1, n, \delta)$ is a birational linear projection of $v_{\delta}\left(\mathbb{P}^{r+1}\right)$ from a linear space of dimension $\bar{\pi}(r, 2, \delta)-\bar{\pi}(r, n, \delta)-1$.

The preceding statement concerns varieties $X$ that are $n$-covered by irreducible curves of degree $\delta$ with respect to an arbitrary given Cartier divisor, hence it is more general than the corresponding result in [52] considering embedded $X=\bar{X}(r+$ $1, n, \delta) \subset \mathbb{P}^{\bar{\pi}(r, n, \delta)-1}$. Note however that the main tool used to prove Theorem 2.4, namely the reduction to the well understood case $n=2$ via an osculating projection, is the same as in [52].

\section{Bound for the top self intersection of a nef divisor}

In this section, as a consequence of part (i) of Theorem 2.4, we prove a bound for the top self intersection of a nef divisor $D$ on a proper variety $X$ such that through $n \geq 2$ general points there passes an irreducible curve $C$ with $(D \cdot C)=\delta \geq n-1$. In particular we obtain a bound for the degree of varieties $X(r+1, n, \delta) \subset \mathbb{P}^{N}$. The bound (3.1) below generalizes a result usually attributed to Fano, who proved it for $n=2$. The reader can consult the modern reference [35], Proposition V.2.9, for the case $n=2$ of Fano's result and also the several applications given in loc. cit., e.g. to the boundedness of the number of components of families of smooth Fano varieties of a fixed dimension, see [35], Chapter V.

Theorem 3.1. Let $X$ be a proper irreducible variety of dimension $r+1$, let $D$ be a nef Cartier divisor on $X$ and suppose that through $n \geq 2$ general points there passes an irreducible curve $C$ such that $(D \cdot C)=\delta \geq n-1$. Then

$$
D^{r+1} \leq \frac{\delta^{r+1}}{(n-1)^{r}} .
$$

In particular, if $X=X(r+1, n, \delta) \subset \mathbb{P}^{N}$, then

$$
\operatorname{deg}(X) \leq \frac{\delta^{r+1}}{(n-1)^{r}} .
$$


Proof. By the Asymptotic Riemann-Roch Theorem, see for example [35], Theorem VI.2.15, we know that

$$
h^{0}\left(\mathcal{O}_{X}(\ell D)\right)=D^{r+1} \frac{\ell^{r+1}}{(r+1) !}+O\left(\ell^{r}\right)
$$

so that

$$
D^{r+1}=\lim _{\ell \rightarrow+\infty} \frac{(r+1) ! h^{0}\left(\mathcal{O}_{X}(\ell D)\right)}{\ell^{r+1}} .
$$

Since $X$ is $n$-covered by a family of irreducible curves having intersection with $D$ equal to $\delta, X$ is also $n$-covered by a family of irreducible curves having intersection $\delta \ell$ with $\ell D$ for any $\ell>0$. Theorem 2.4 yields

$$
h^{0}\left(\mathcal{O}_{X}(\ell D)\right) \leq \bar{\pi}(r, n, \delta \ell)
$$

for every positive integer $\ell$. From (3.3) we deduce

$$
D^{r+1} \leq \liminf _{\ell \rightarrow+\infty} \frac{(r+1) ! \bar{\pi}(r, n, \delta \ell)}{\ell^{r+1}} .
$$

Let $\rho_{\ell}=\left\lfloor\frac{\delta \ell}{n-1}\right\rfloor$ for $\ell>0$. The definition of $\bar{\pi}(r, n, \delta \ell)$ in (1.3) implies that

$$
D^{r+1} \leq \liminf _{\ell \rightarrow+\infty} \frac{(n-1)\left(r+1+\rho_{\ell}\right) !}{\ell^{r+1} \rho_{\ell} !} .
$$

Using Stirling's formula, for $\ell \rightarrow+\infty$, we have

$$
\begin{aligned}
\frac{(n-1)\left(r+1+\rho_{\ell}\right) !}{\ell^{r+1} \rho_{\ell} !} & \sim \frac{(n-1) \sqrt{r+1+\rho_{\ell}}\left(\frac{r+1+\rho_{\ell}}{e}\right)^{r+1+\rho_{\ell}}}{\ell^{r+1} \sqrt{\rho_{\ell}}\left(\frac{\rho_{\ell}}{e}\right)^{\rho_{\ell}}} \\
& \sim \frac{(n-1)\left(r+1+\rho_{\ell}\right)^{r+1}}{\ell^{r+1} e^{r+1}}\left(1+\frac{r+1}{\rho_{\ell}}\right)^{\rho_{\ell}} .
\end{aligned}
$$

Since $\rho_{\ell} \rightarrow+\infty$ if $\ell \rightarrow+\infty$ and recalling that $\lim _{x \rightarrow+\infty}\left(1+\frac{r+1}{x}\right)^{x}=e^{r+1}$, we obtain

$$
\frac{(n-1)\left(r+1+\rho_{\ell}\right) !}{\ell^{r+1} \rho_{\ell} !} \sim \frac{(n-1) \rho_{\ell}^{r+1}}{\ell^{r+1}} .
$$

But $\rho_{\ell} \sim \frac{\delta \ell}{n-1}$ if $\ell \rightarrow+\infty$ hence we finally get

$$
D^{r+1} \leq \liminf _{\ell \rightarrow+\infty} \frac{(n-1)\left(\frac{\delta \ell}{n-1}\right)^{r+1}}{\ell^{r+1}}=\frac{\delta^{r+1}}{(n-1)^{r}} .
$$


Remark 3.2. The bound (3.2) is sharp for $n=2$ since $\delta^{r+1}$ is the degree of $v_{\delta}\left(\mathbb{P}^{r+1}\right)$. More generally, it is sharp for every $n \geq 2$ as soon as $\delta=\rho(n-1)$ for some integer $\rho$ since in this case $\delta^{r+1} /(n-1)^{r}=\rho^{r+1}(n-1)$ is the degree of $v_{\rho}(Y)$ for any non-degenerate variety $Y^{r+1} \subset \mathbb{P}^{n+r-1}$ of minimal degree $n-1$.

We apply the previous bound on the degree to classify the $\bar{X}(r+1, n, n-1+k)$ 's for $n$ sufficiently large, when $r$ and $k$ are fixed.

Suppose $k=0$. Since $\bar{\pi}(r, n, n-1)=n+r, X=\bar{X}(r+1, n, n-1) \subset \mathbb{P}^{n+r-1}$ is a variety of minimal degree equal to $\operatorname{codim}(X)+1=n-1$ by (3.2), as it is well known. These varieties were classified in ancient times by classical algebraic geometers, see [21] and also Section 4.

Now we consider the case $k>0$. When $n$ is sufficiently large, we have $m=k+1$, $m^{\prime}=n-k-2$ and $\rho=1$, yielding $\bar{\pi}(r, n, n-1+k)=(k+1)(r+2)+n-k-2$. So (3.2) implies that $X=\bar{X}(r+1, n, n-1+k)$ is a variety of minimal degree as soon as the quantity

$$
\theta=(n-1+k)^{r+1}-(n-1)^{r}(n+k(r+1)-2)
$$

is strictly positive. But $\theta=n^{r}+O\left(n^{r-1}\right)$ as a simple and direct expansion shows. Therefore for $n$ sufficiently large $\theta>0$ and $X=\bar{X}(r+1, n, n-1+k)$ is a variety of minimal degree. Assuming moreover that $n>5$, as we shall do from now on, one deduces that $X$ is a rational normal scroll $S_{a_{0}, \ldots, a_{r}}$ with $0 \leq a_{0} \leq \cdots \leq a_{r}$ and $\sum_{i=0}^{r} a_{i}=n+k(r+1)-1$. We want to prove that $X$ is projectively equivalent to $S_{\alpha_{0}+k, \ldots, \alpha_{r}+k}$ with $\alpha_{0}, \ldots, \alpha_{r}$ verifying $0 \leq \alpha_{0} \leq \cdots \leq \alpha_{r}$ and $\sum_{i=0}^{r} \alpha_{i}=$ $n-1$. With the terminology introduced in Section 4 this will mean exactly that $X=\bar{X}(r+1, n, n-1+k)$ is of Castelnuovo type for $n$ sufficiently large.

Assume that $x_{1}, \ldots, x_{k+1}$ and $y_{1}, \ldots, y_{n-k-2}$ are general points of $X$ and let $\pi=\bar{\pi}(r, n, n-1+k)$. According to (1.6),

$$
\mathbb{P}^{\pi-1}=\langle X\rangle=\left(\bigoplus_{i=1}^{k+1} \operatorname{Osc}_{X}^{1}\left(x_{i}\right)\right) \oplus\left\langle y_{1}, \ldots, y_{n-k-2}\right\rangle .
$$

Let us introduce some notation. Let $0 \leq a_{0} \leq a_{1} \leq \cdots \leq a_{r}$ with $a_{r}>$ 0 be integers and set $\mathbb{P}\left(a_{0}, \ldots, a_{r}\right):=\mathbb{P}\left(\bigoplus_{i=0}^{r} \mathcal{O}_{\mathbb{P}^{1}}\left(a_{i}\right)\right)$. Let $H$ be a divisor in $\left|\mathcal{O}_{\mathbb{P}\left(a_{0}, \ldots, a_{r}\right)}(1)\right|$ and consider the morphism $\phi=\phi_{|H|}: \mathbb{P}\left(a_{1}, \ldots, a_{n}\right) \rightarrow$ $\mathbb{P} a_{0}+\cdots+a_{r}+r$ whose image is denoted $S_{a_{0}, \ldots, a_{r}}$ and called a rational normal scroll of type $\left(a_{0}, \ldots, a_{r}\right)$. The morphism $\phi$ is birational onto its image so that $S_{a_{0}, \ldots, a_{r}}$ has dimension $r+1$ and its degree is $a_{0}+\cdots+a_{r}$. The scroll $S_{a_{0}, \ldots, a_{r}}$ is smooth if and only if $a_{0}>0$ and $\phi$ is an embedding in this case. If $0=a_{i}<a_{i+1}$, then $S_{a_{0}, \ldots, a_{r}}$ is a cone over $S_{a_{i+1}, \ldots, a_{r}}$ with vertex a $\mathbb{P}^{i}$.

Lemma 3.3 ([16], p. 13). Let $b_{0}, \ldots, b_{r}$ be natural integers such that $0=b_{0}=$ $\cdots=b_{i}<b_{i+1} \leq b_{i+2} \leq \cdots \leq b_{r}$, let $\left\{d_{1}^{\prime}, \ldots, d_{r-i}^{\prime}\right\}=\left\{b_{i+1}-1, \ldots, b_{r}-2\right\}$ 
and let $\left\{d_{1}, \ldots, d_{r-i}\right\}$ be a rearrangement of $\left\{d_{1}^{\prime}, \ldots, d_{r-i}^{\prime}\right\}$ such that $0 \leq d_{1} \leq$ $d_{2} \leq \cdots \leq d_{r-i}$. Then the general tangential projection of $S_{b_{0}, \ldots, b_{r}}$ is $S_{d_{1}, \ldots, d_{r-i}}$.

Thus if $a_{0}<k$, there would exist $\ell<k+1$ such that the image $X^{\prime}$ of $X$ via the linear projection $p_{S}$ from $S=\operatorname{Osc}_{X}^{1}\left(x_{1}\right) \oplus \cdots \oplus \operatorname{Osc}_{X}^{1}\left(x_{\ell}\right)$ would be a rational normal scroll of dimension $r^{\prime} \leq r$. This would imply $\operatorname{dim}(\langle\bar{X}(r+1, n, n-1+k)\rangle)<$ $\bar{\pi}(r, n, n-1+k)-1$, leading to a contradiction. In conclusion we proved the following consequence of (3.2).

Corollary 3.4. If $n$ is sufficiently large, a variety $\bar{X}(r+1, n, n-1+k)$ is projectively equivalent to a rational normal scroll $S_{\alpha_{0}+k, \ldots, \alpha_{r}+k}$ with $\alpha_{0}, \ldots, \alpha_{r}$ such that $\sum_{i=0}^{r} \alpha_{i}=n-1$.

Remark 3.5. The value of $n$ in the previous result can be made effective: $\bar{X}(r+$ $1, n, n-1+k)$ is a scroll as in the previous corollary as soon as $n \geq \max (6, k-2)$ and $\theta>0$, where $\theta$ is the quantity defined in (3.5).

\section{Some examples of varieties $\bar{X}(r+1, n, \delta)$}

In this section we describe in detail three classes of $\bar{X}(r+1, n, \delta)$. The simplest ones are the so-called varieties of minimal degree. Next, using the theory of Castelnuovo varieties, we will construct examples of $\bar{X}(r+1, n, \delta)$ for arbitrary $n, r$ and $\delta \geq n-1$. These latter examples have already been presented in [52]. Finally, we will show that twisted cubic curves associated to Jordan algebras of rank 3 are examples of $\bar{X}(r+1,3,3)$.

4.1. Varieties of minimal degree and their associated models [21], [30]. It is wellknown (see [28], p. 173) that the degree of an irreducible non-degenerate projective variety $Y \subset \mathbb{P}^{n+r-1}$ of dimension $r+1$ satisfies $\operatorname{deg}(Y) \geq \operatorname{codim}(Y)+1=n-1$. By definition, $Y \subset \mathbb{P}^{n+r-1}$ is a variety of minimal degree if its degree is $n-1$. Such varieties exist and are well known. Their classification goes back to Bertini and Enriques and can be summarized as follows (the notation being as in [30]):

Theorem 4.1. The following is an exhaustive non redundant list of the $(r+1)$ dimensional varieties $Y \subset \mathbb{P}^{n+r-1}$ of minimal degree $n-1$ :

(1) the rational normal scrolls $S_{a_{0}, \ldots, a_{r}}$ for some integers $a_{0}, \ldots, a_{r}$ verifying $0 \leq$ $a_{0} \leq a_{1} \leq \cdots \leq a_{r} \leq n-1$ and $a_{0}+\cdots+a_{r}=n-1$;

(2) the ambient space $\mathbb{P}^{r+1}$ itself $(n=2)$;

(3) the quadric hypersurfaces of rank $\varrho \geq 5(n=3)$;

(4) the cones over a Veronese surface in $\mathbb{P}^{5}(n=5)$. 
Recall that a scroll $S_{a_{0}, \ldots, a_{r}}$ is singular if and only if $a_{0}=0$. In this case, it is a cone over the scroll $S_{a_{j}, \ldots, a_{r}}$ where $j$ stands for the smallest integer such that $a_{j} \neq 0$.

Let $Y \subset \mathbb{P}^{n+r-1}$ be a variety of minimal degree. The space spanned by $n$ generic distinct points $y_{1}, \ldots, y_{n}$ on $Y$ is an $(n-1)$-dimensional subspace in $\mathbb{P}^{n+r-1}$. The latter being generic, it intersects $Y$ along an irreducible non-degenerate curve of degree $n-1$, which by Lemma 2.3 is a rational normal curve in $\left\langle y_{1}, \ldots, y_{n}\right\rangle$ passing through $y_{i}$ for any $i=1, \ldots, n$. This shows that $Y \subset \mathbb{P}^{n+r-1}$ is $n$-covered by rational normal curves of degree $n-1$. Since $\bar{\pi}(r, n, n-1)=n+r$, it follows that varieties of minimal degree are examples of $\bar{X}(r+1, n, n-1)$, which will be called models of minimal degree. Note that also the converse is true because according to Theorem 3.1, every $\bar{X}(r+1, n, n-1) \subset \mathbb{P}^{n+r-1}$ is a variety of minimal degree $n-1$.

4.2. Castelnuovo's varieties and their associated models [30], [14], [52]. Let $V \subset \mathbb{P}^{n+r-1}$ be an irreducible non-degenerate variety of dimension $r$ and degree $d>1$. There is an explicit bound on the geometric genus $g(V)$ of $V$ in terms of an explicit constant depending on $d, n$ and $r$. The geometric genus $g(V)$ is defined as the dimension $h^{0}\left(K_{\tilde{V}}\right)$ for one (hence for all) resolution of the singularities $\widetilde{V} \rightarrow V$ of $V$.

Theorem 4.2 (Castelnuovo-Harris bound [30]). The following bound

$$
g(V) \leq \pi(r, n, d)
$$

holds for the geometric genus of $V \subset \mathbb{P}^{n+r-1}$. In particular $g(V)=0$ if $d<$ $r(n-1)+2$.

An irreducible variety $V \subset \mathbb{P}^{n+r-1}$ as above and such that $g(V)=\pi(r, n, d)>0$ is called a Castelnuovo variety. Note that in this case necessarily $d \geq r(n-1)+2$.

Remark 4.3. The bound (4.1) can be generalized to more general objects than projective varieties. Indeed, a basic result of web geometry says that the bound $\operatorname{rk}(W) \leq$ $\pi(r, n, d)$ holds for the rank $\operatorname{rk}(W)$ of an $r$-codimensional $d$-web $W=W_{d}(n, r)$ defined on a manifold of dimension $n r$. This result, due to Chern and Griffiths [13], can be proved by quite elementary methods (see [51] and [49]). Combined with Abel's addition theorem, this implies the inequality $h^{0}\left(V, \omega_{V}\right) \leq \pi(r, n, d)-$ here $\omega_{V}$ denotes the sheaf of abelian differential $r$-forms on $V$, see [37], [43], [3], [31] as soon as $V$ is such that its generic 0 -dimensional linear section is in general position in its span, which is stronger than (4.1).

The classification of projective curves of maximal genus has been obtained by Castelnuovo in 1889. More recently, in [30], Harris proved the following result. 
Proposition 4.4. Let $V \subset \mathbb{P}^{n+r-1}$ be a Castelnuovo variety of dimension $r \geq 1$ and codimension at least 2 . The linear system $\left|\mathcal{I}_{V}(2)\right|$ cuts out a variety of minimal degree $Y \subset \mathbb{P}^{n+r-1}$ of dimension $r+1$.

Thus a Castelnuovo variety $V \subset \mathbb{P}^{n+r-1}$ of dimension $r$ is a divisor in the variety of minimal degree $Y \subset \mathbb{P}^{n+r-1}$ cut out by $\left|\mathcal{I}_{V}(2)\right|$. This property was used by Harris to describe Castelnuovo varieties (see also the refinements by Ciliberto in [14]): if $p: \widetilde{Y} \rightarrow Y$ denotes a desingularization (obtained for instance by blowing-up the vertex of the cone $Y$ when it is singular), Harris determines the class $[\widetilde{V}]$ of $\widetilde{V}$ (the strict transform of $V$ in $\widetilde{Y}$ via $p$ ) in the Picard group of $\widetilde{Y}$. Assuming (to simplify) that $\widetilde{V}$ is smooth, let $L_{V}=K_{\tilde{Y}}+\widetilde{V}$. By adjunction theory, there is a short exact sequence of sheaves

$$
0 \rightarrow K_{\tilde{Y}} \rightarrow K_{\tilde{Y}}(\tilde{V}) \rightarrow K_{\widetilde{V}} \rightarrow 0 .
$$

Since $h^{0}\left(\tilde{Y}, K_{\tilde{Y}}\right)=h^{1}\left(\tilde{Y}, K_{\tilde{Y}}\right)=0$ (because $\tilde{Y}$ is smooth and rational), the map

$$
H^{0}\left(\tilde{Y}, L_{V}\right) \rightarrow H^{0}\left(\tilde{V}, K_{\tilde{V}}\right)
$$

is an isomorphism. Thus it induces rational maps $\phi_{V}=\phi_{\left|K_{V}\right|}$ and $\Phi_{V}=\Phi_{\left|L_{V}\right|^{\circ}} p^{-1}$ such that the following diagram of rational maps is commutative:

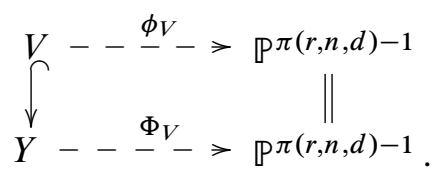

Let $X_{V}$ be (the closure of) the image of $\Phi_{V}$. It is an irreducible non-degenerate subvariety in $\mathbb{P}^{\pi(r, n, d)-1}$ and $\operatorname{dim}\left(X_{V}\right)=\operatorname{dim}(Y)=r+1$. Moreover, one proves that the image by $\Phi_{V}$ of a generic 1-dimensional linear section of $Y$, that is of a rational normal curve of degree $n-1$ passing through $n$ general points of $Y$, is a rational normal curve of degree $\delta=d-r(n-1)-2$ contained in $X_{V}$. Thus $X_{V} \subset \mathbb{P}^{\pi(r, n, d)-1}=\mathbb{P}^{\bar{\pi}(r, n, \delta)-1}$ is an example of $\bar{X}(r+1, n, \delta)$. These examples will be called models associated to the Castelnuovo variety $V \subset \mathbb{P}^{n+r-1}$ and also models of Castelnuovo type. They are described in detail in [52] where the authors also prove the following result.

Theorem 4.5. Let $X \in \bar{X}(r+1, n, \delta)$. If $\delta \neq 2 n-3$ then $X$ is of Castelnuovo type.

It follows that varieties $\bar{X}(r+1, n, \delta)$ not of Castelnuovo type can exists only for $\delta=2 n-3$. In the sequel, we shall focus on the case $n=3$ and present some examples of varieties $\bar{X}(r+1,3,3)$ not of Castelnuovo constructed from Jordan algebras. 
4.3. Models of Jordan type associated to cubic Jordan algebras. Recall that a (complex) Jordan algebra is a $\mathbb{C}$-vector space $\mathbb{J}$ with a $\mathbb{C}$-bilinear product $\mathbb{J} \times \mathbb{J} \rightarrow \mathbb{J}$ verifying

$$
x^{2}(y x)=\left(x^{2} y\right) x \quad \text { for all } x, y \in \mathbb{J} .
$$

We will restrict here to the case of commutative Jordan algebras of finite dimension admitting a unit, denoted by $e$. A classical result in this theory ensures that a Jordan algebra is power-associative: for every $x \in \mathbb{J}$ and any $k \in \mathbb{N}$, the $k$ th-power $x^{k}$ of $x$ is well-defined. This allows us to define the rank of $\mathbb{J}$, denoted by $\operatorname{rk}(\mathbb{J})$ : it is the dimension (as a complex vector space) of the subalgebra $\mathbb{C}[x]=\operatorname{Span}_{\mathbb{C}}\left\langle x^{k} \mid k \in \mathbb{N}\right\rangle$ generated by a general element $x \in \mathbb{J}$. Let $m=\operatorname{rk}(\mathbb{J})$ and let $\ell_{x}$ be the restriction of the multiplication by $x$ to $\mathbb{C}[x]$ and let $M_{x}$ be its associated minimal polynomial. Then the relation $M_{x}\left(\ell_{x}\right) e=\mathbf{0}$ expands to

$$
x^{m}-\sigma_{1}(x) x^{m-1}+\cdots+(-1)^{m} \sigma_{m}(x) e=\mathbf{0},
$$

where $x \mapsto \sigma_{i}(x)$ is a homogeneous polynomial map of degree $i$ on $\mathbb{J}$ (for every $i=1, \ldots, m)$. By definition, (4.4) is the generic minimum polynomial of the Jordan algebra $\mathbb{J}$ (cf. [24], Proposition II.2.1). Its generic trace is the linear map $T: x \mapsto$ $T(x)=\sigma_{1}(x)$ and the homogeneous polynomial map $N: x \mapsto N(x)=\sigma_{m}(x)$ of degree $m$ is its generic norm. The latter is multiplicative in the following sense: for all $y \in \mathbb{J}$, we have $N\left(x x^{\prime}\right)=N(x) N\left(x^{\prime}\right)$ for every $x, x^{\prime} \in \mathbb{C}[y]$ (see Proposition II.2.2 of [24]).

One defines the adjoint $x^{\#}$ of an element $x \in \mathbb{J}$ by setting

$$
x^{\#}=\sum_{i=0}^{m-1} \sigma_{i}(x)(-x)^{m-1-i}
$$

where $\sigma_{0}=1$. It follows from (4.4) that $x x^{\#}=x^{\#} x=N(x) e$ so that $N(x) \neq 0$ implies that $x$ is invertible (for the Jordan product) with inverse $x^{-1}=N(x)^{-1} x^{\#}$.

The map $i: x \mapsto x^{-1}$ is a birational involution on $\mathbb{J}$. Let $\operatorname{Str}(\mathbb{J})$ be the set of $g \in \mathrm{GL}(\mathbb{J})$ such that $g \circ i \equiv i \circ h$ (as birational maps on $\mathbb{J}$ ) for a certain $h \in \mathrm{GL}(\mathbb{J}$ ). When it exists, such a $h$ is unique: by definition, it is the adjoint of $g$ and is denoted by $g^{\#}$. One proves (see [61]) that $\operatorname{Str}(\mathbb{J})$ is a closed algebraic subgroup of $G L(\mathbb{J})$, called the structural group of the Jordan algebra $\mathbb{J}$. Moreover, $g \mapsto g^{\#}$ is an automorphism of $\operatorname{Str}(\mathbb{J})$ and there exists a character $\eta: \operatorname{Str}(\mathbb{J}) \rightarrow \mathbb{C}^{*}$ such that $N(g(x))=\eta_{g} N(x)$ for all $x \in \mathbb{J}(c f$. [61], Proposition 1.5).

The complex vector space $\mathbb{Z}_{2}(\mathbb{J})$ of Zorn's matrices is defined by

$$
\mathcal{Z}_{2}(\mathbb{J})=\left\{\left(\begin{array}{ll}
s & x \\
y & t
\end{array}\right) \mid s, t \in \mathbb{C}, x, y \in \mathbb{J}\right\} .
$$

Assuming from now on that $\mathbb{I}$ is of rank 3 , one defines the twisted cubic associated to $\mathbb{J}$ (noted by $X_{\mathbb{J}}^{3}$ ) as the (Zariski)-closure in $\mathbb{P} \mathcal{Z}_{2}(\mathbb{J})$ of the image of the polynomial 
affine embedding

$$
\begin{aligned}
v_{3}: \mathbb{J} & \longrightarrow \mathbb{P} \mathcal{Z}_{2}(\mathbb{J}), \\
x & \longmapsto\left[\begin{array}{cc}
1 & x \\
x^{\#} & N(x)
\end{array}\right] .
\end{aligned}
$$

Then the Zariski-closure of $v_{3}(\mathbb{C} e)$ in $\mathbb{P} Z_{2}(\mathbb{J})$ is a rational normal curve of degree 3 included in $X_{\mathbb{J}}^{3}$ that we denote by $C_{\mathbb{J}}$ :

$$
C_{\mathbb{J}}=\left\{\left[\begin{array}{cc}
1 & t e \\
t^{2} e & t^{3}
\end{array}\right] \mid t \in \mathbb{C}\right\} \cup\left\{\left[\begin{array}{ll}
0 & 0 \\
0 & 1
\end{array}\right]\right\} .
$$

It contains the following three points:

$$
0_{\mathbb{J}}=v_{3}(0)=\left[\begin{array}{ll}
1 & 0 \\
0 & 0
\end{array}\right], \quad 1_{\mathbb{J}}=v_{3}(e)=\left[\begin{array}{ll}
1 & e \\
e & 1
\end{array}\right] \quad \text { and } \quad \infty_{\mathbb{J}}=\left[\begin{array}{ll}
0 & 0 \\
0 & 1
\end{array}\right] .
$$

Recall that $i$ stands for the inverse map $x \mapsto x^{-1}$. From now on we shall suppose $g \in \operatorname{Str}(\mathbb{J})$. For a fixed $\omega \in \mathbb{J}$, let $t_{\omega}: \mathbb{J} \rightarrow \mathbb{J}$ be the translation $x \mapsto x+\omega$ and denote by \# the bilinear map associated to $x^{\#}$ : one has $x \# y=(x+y)^{\#}-x^{\#}-y^{\#}$ for $x, y \in \mathbb{J}$.

For $\omega \in \mathbb{J}$ and $g \in \operatorname{Str}(\mathbb{J})$, one sets for every $M=\left[\begin{array}{cc}s & x \\ y & t\end{array}\right] \in \mathbb{P} Z_{2}(\mathbb{J})$ :

$$
\begin{aligned}
I(M) & =\left[\begin{array}{ll}
t & y \\
x & s
\end{array}\right], \\
G_{g}(M) & =\left[\begin{array}{cc}
s & g(x) \\
\eta_{g} g^{\#}(y) & \eta_{g} t
\end{array}\right]
\end{aligned}
$$

and

$$
T_{\omega}(M)=\left[\begin{array}{cc}
s & x+s \omega \\
y+\omega \# x+s \omega^{\#} & t+T(y \omega)+T\left(x \omega^{\#}\right)+s N(\omega)
\end{array}\right] .
$$

These maps are projective automorphisms of $\mathbb{P} Z_{2}(\mathbb{J})$.

The proof of the following lemma is straightforward and left to the reader.

Lemma 4.6. For every $\omega \in \mathbb{J}$ and for every $g \in \operatorname{Str}(\mathbb{J})$, we have

$$
v_{3} \circ i=I \circ v_{3}, \quad v_{3} \circ g=G_{g} \circ v_{3} \quad \text { and } \quad v_{3} \circ t_{\omega}=T_{\omega} \circ v_{3} .
$$

Consequently, the maps $I, G$ and $T_{\omega}$ are projective automorphisms of the cubic $X_{\mathbb{J}}^{3}$.

Let $\operatorname{Conf}(\mathbb{J})$ be the conformal group of $\mathbb{J}$, that is the subgroup of PGL $\left(Z_{2}(\mathbb{J})\right)$ generated by $I$ and the maps $G_{g}$ and $T_{\omega}$ for all $\omega \in \mathbb{J}$ and all $g \in \operatorname{Str}(\mathbb{J})$. From Lemma 4.6, it follows that $\operatorname{Conf}(\mathbb{J})$ is a subgroup of the group of projective automorphisms of $X_{\mathbb{J}}^{3}$. 
Proposition 4.7. The group of projective automorphisms of $X_{\mathbb{J}}^{3}$ acts transitively on 3-uple's of general points of $X_{\mathbb{J}}^{3}$. In fact, if $x_{1}, x_{2}, x_{3} \in \mathbb{J}$ are sufficiently general, there exists $\mu \in \operatorname{Conf}(\mathbb{I})$ such that $\mu\left(v_{3}\left(x_{1}\right)\right)=0_{\mathbb{J}}, \mu\left(v_{3}\left(x_{2}\right)\right)=1_{\mathbb{J}}$ and $\mu\left(v_{3}\left(x_{3}\right)\right)=\infty_{\mathbb{J}}$.

Proof. First let $\mathcal{G}=\operatorname{Str}(\mathbb{J}) \cdot e$ be the orbit in $\mathbb{J}$ of the unit $e$ under the linear action of the structural group. By Theorem 6.5 of [61], one knows that $\mathcal{G}$ is a Zariski-open subset of $\mathbb{J}$.

Assume that $x, y, z \in \mathbb{J}$ are such that (1) $y_{1}=y-x$ and $z_{1}=z-x$ are invertible; (2) $z_{2}=z_{1}^{-1}-y_{1}^{-1} \in \mathcal{G}$, i.e $z_{2}=g(e)$ for a certain $g \in \operatorname{Str}(\mathbb{J})$. Then set

$$
\mu=\left(G_{g}\right)^{-1} \circ T_{-\left(y_{1}\right)^{-1}} \circ I \circ T_{-x} \in \operatorname{Conf}(\mathbb{J}) .
$$

We leave to the reader to verify that $\mu\left(v_{3}(x)\right)=\infty_{\mathbb{J}}, \mu\left(v_{3}(y)\right)=0_{\mathbb{J}}$ and $\mu\left(v_{3}(z)\right)=$ $1_{\mathbb{J}}$. Since $v_{3}(\mathbb{J})$ is dense in $X_{\mathbb{J}}^{3}$, the conclusion follows.

Let $x_{1}, x_{2}, x_{3} \in \mathbb{J}$ and let $\mu \in \operatorname{Conf}(\mathbb{J})$ be as in the statement of Proposition 4.7. Since $\mu \in \operatorname{PGL}\left(\mathcal{Z}_{2}(\mathbb{J})\right)$, the curve $\mu^{-1}\left(C_{\mathbb{J}}\right)$ is a rational normal curve of degree 3 passing through the points $\nu_{3}\left(x_{i}\right)$ for $i=1,2,3$. Since $\mu$ is a projective automorphism of $X_{\mathbb{J}}^{3}$ (by Lemma 4.6 above), this twisted cubic curve is also contained in $X_{\mathbb{J}}^{3}$. Thus we have proved the following result.

Corollary 4.8. The twisted cubic $X_{\mathbb{J}}^{3}$ associated to a rank 3 Jordan algebra $\mathbb{J}$ is 3-covered by rational normal curves of degree 3 .

Let $k$ be the dimension of a rank 3 Jordan algebra $\mathbb{J}$. Then $X_{\mathbb{J}}^{3}$ is a non-degenerate algebraic subvariety of the projective space $\mathbb{P} Z_{2}(\mathbb{J})$, whose dimension is $2 k+1$. Since $\pi(k, 3,3)=2 k+2$, one obtains that $X_{\mathbb{J}}^{3} \subset \mathbb{P} Z_{2}(\mathbb{J})=\mathbb{P}^{2 k+1}$ is an example of $\bar{X}(k, 3,3)$. Thus the cubics $X_{\mathbb{J}}^{3}$ associated to rank 3 Jordan algebras are examples of varieties of type $\bar{X}(k, 3,3)$, which will be called models of Jordan type. A consequence of Theorem 5.2 is that a $X_{\mathbb{J}}^{3}$ is never of Castelnuovo type.

In fact, using Theorem 5.2, one can easily prove the following criterion which characterizes the varieties $\bar{X}(r+1, n, 2 n-3)$ of Castelnuovo type for arbitrary $n \geq 3$.

Proposition 4.9. A variety $X=\bar{X}(r+1, n, 2 n-3)$ is of Castelnuovo type if and only if for any $n-2$ general point $x_{2}, \ldots, x_{n-1} \in X$, the intersection of the space $\bigoplus_{i=2}^{n-1} \operatorname{Osc}_{X}^{1}\left(x_{i}\right)$ with $X$ contains a hypersurface.

In fact, it can be verified that the $X=\bar{X}(r+1, n, 2 n-3)$ of Castelnuovo type are exactly the two scrolls $S_{n-2, \ldots, n-2, n}$ and $S_{n-2, \ldots, n-2, n-1, n-1}$ in $\mathbb{P}^{(n-1)(r+2)-1}$. 
4.3.1. Examples. We shall describe some explicit examples of models of Jordan type.

A Jordan algebra $\mathbb{J}$ is simple if it does not admit proper non-trivial ideals. It is semi-simple if it is a direct product of simple Jordan algebras (or equivalently, if the bilinear symmetric form $(x, y) \mapsto T(x y)$ is non-degenerate, see Theorem 5, p. 240, in [34]).

Exemple 4.10. Let $B$ be a symmetric bilinear form on $W=\mathbb{C}^{r-1}$. Then $\mathbb{J}^{\prime}=\mathbb{C} \oplus W$ with the product defined by

$$
(\lambda, y) \diamond\left(\lambda^{\prime}, y^{\prime}\right)=\left(\lambda \lambda^{\prime}-B\left(y, y^{\prime}\right), \lambda y^{\prime}+\lambda^{\prime} y\right)
$$

is a Jordan algebra of rank 2. Moreover, $x^{2}-2 \lambda x+\left(\lambda^{2}+B(y, y)\right) e=0$ for any $x=(\lambda, y) \in \mathbb{J}^{\prime}$ (where $e=(1,0)$ ). The generic norm and the generic trace are $N(\lambda, y)=\lambda^{2}+B(y, y)$ and $T(\lambda, y)=2 \lambda$, respectively, hence the adjoint is given by $x^{\#}=(\lambda,-y)$. One verifies that $\mathbb{C} \oplus W$ is semi-simple if and only if $B$ is non-degenerate and $\mathbb{C} \oplus W$ is simple if $B$ is non-degenerate and $r>2$. When $r=2, \mathbb{J}^{\prime}=\mathbb{C} \oplus W$ with the product (4.6) is isomorphic to the direct product (of Jordan algebras) $\mathbb{C} \times \mathbb{C}$.

One can define the conic associated to a Jordan algebra $\mathbb{J}^{\prime}$ of rank 2. By definition, it is the (Zariski)-closure, denoted by $X_{\mathbb{J}^{\prime}}^{2}$, of the image of the affine map $\mathbb{J}^{\prime} \ni x \mapsto$ $[1: x: N(x)] \in \mathbb{P}\left(\mathbb{C} \oplus \mathbb{J}^{\prime} \oplus \mathbb{C}\right)$. Since $N$ is homogeneous of degree $2, X_{\mathbb{J}^{\prime}}^{2}$ is a non-degenerate quadric hypersurface in $\mathbb{P}^{r+1}$ (where $r=\operatorname{dim}_{\mathbb{C}} \mathbb{J}^{\prime}$ ). It is smooth if and only if $\mathbb{J}^{\prime}$ is simple. In any case, $X_{\mathbb{J}^{\prime}}^{2}$ is an example of $\bar{X}(r, 3,2)$ (of minimal type).

Lemma 4.11. If $\mathbb{J}=\mathbb{C} \times \mathbb{J}^{\prime}$ is the direct product of $\mathbb{C}$ with a Jordan algebra $\mathbb{J}^{\prime}$ of rank 2 and dimension $r$, then $\mathbb{J}$ is of rank 3 and $X_{\mathbb{J}}^{3} \simeq \mathbb{P}^{1} \times X_{\mathbb{J}^{\prime}}^{2}$ Segre embedded in $\mathbb{P}^{2 r+3}$.

This result ensures the existence of examples of models of Jordan type of any dimension. For $\mathbb{J}=\mathbb{C} \times \mathbb{J}^{\prime}$ with $\mathbb{J}^{\prime}$ of rank 2 , note that $X_{\mathbb{J}}^{3}$ is smooth if and only if $\mathbb{J}$ is semi-simple.

\subsubsection{Examples of $\bar{X}(r+1,3,3)$ 's associated to simple Jordan algebras of} rank 3. Let us now consider models of Jordan type associated to simple Jordan algebras of rank 3, which can be completely classified. We recall the well-known Hurwitz Theorem that there are exactly four composition algebras over the field of real numbers: $\mathbb{R}$ itself, the field $\mathbb{C}$ of complex numbers and the algebras $\mathbb{U}$ and $\mathbb{( O )}$ of quaternions and octonions respectively (the reader can consult [2] for a nice introduction to these objects). 
If $\mathbb{A}$ denotes one of these algebras, let $\mathbb{A}_{\mathbb{C}}=\mathbb{A} \otimes \mathbb{C}$ be its complexification (over $\mathbb{R}$ ). Any such $\mathbb{A}_{\mathbb{C}}$ is a complex composition algebra: for $x_{i}=a_{i} \otimes r_{i} \in \mathbb{A}_{\mathbb{C}}$, with $a_{i} \in \mathbb{A}$ and $r_{i} \in \mathbb{C}$ for $i=1,2$, we define $x_{1} \cdot x_{2}=a_{1} a_{2} \otimes r_{1} r_{2}, \overline{x_{1}}=\overline{a_{1}} \otimes r_{1}$ and $\left\|x_{1}\right\|^{2}=x_{1} \cdot \overline{x_{1}} \in \mathbb{C}$. Of course, except for $\mathbb{O}_{\mathbb{C}}$, there are classical isomorphisms (of complex algebras)

$$
\mathbb{R}_{\mathbb{C}} \simeq \mathbb{C}, \quad \mathbb{C}_{\mathbb{C}} \simeq \mathbb{C} \oplus \mathbb{C} \text { and } \mathbb{M}_{\mathbb{C}} \simeq M_{2}(\mathbb{C})
$$

Let $\mathcal{H}_{3}\left(\mathbb{A}_{\mathbb{C}}\right)$ be the space of Hermitian matrices of order three with coefficients in $\mathbb{A}_{\mathbb{C}}$ :

$$
\mathcal{H}_{3}\left(\mathbb{A}_{\mathbb{C}}\right)=\left\{\left(\begin{array}{lll}
r_{1} & \overline{x_{3}} & \overline{x_{2}} \\
x_{3} & r_{2} & \overline{x_{1}} \\
x_{2} & x_{1} & r_{3}
\end{array}\right) \mid x_{1}, x_{2}, x_{3} \in \mathbb{A}_{\mathbb{C}}, r_{1}, r_{2}, r_{3} \in \mathbb{C}\right\} .
$$

The multiplication

$$
(M, N) \mapsto \frac{1}{2}(M N+N M)
$$

induces on $\mathcal{H}_{3}\left(\mathbb{A}_{\mathbb{C}}\right)$ a structure of complex (unital commutative) Jordan algebra. For $\mathbb{A}=\mathbb{R}, \mathbb{C}$ or $\mathbb{Q}$, it is a direct consequence of the fact that $\mathbb{A}$ and hence the rings of $3 \times 3$ matrices, $M_{3}\left(\mathbb{A}_{\mathbb{C}}\right)$, are associative algebras. For $M_{3}\left(\mathbb{O}_{\mathbb{C}}\right)$ a particular argument is needed and we refer to [24], Chapters V and VIII, for this case. One proves (see again [24]) that any Jordan algebra $\mathcal{H}_{3}\left(\mathbb{A}_{\mathbb{C}}\right)$ is simple and of rank 3.

We are now able to describe all simple Jordan algebras of rank three. Their classification is classical (cf. [34], p. 233, or [24] for instance) and is given in Table 1 below with a description of the corresponding cubic curves, see also [46], [39], [18]. The table also shows classical isomorphisms of $\mathcal{H}_{3}\left(\mathbb{A}_{\mathbb{C}}\right)$ with some matrix algebras having (4.7) as Jordan product (in the case $\mathbb{A}=\mathbb{R}, \mathbb{C}$ or $\mathbb{U}$ ).

Table 1. Simple (unital and finite-dimensional) Jordan algebras of rank 3 and their associated cubic curves.

\begin{tabular}{|l|c|}
\hline Jordan algebra $\mathbb{J}$ & Twisted cubic curve $X_{\mathbb{J}}^{3}$ over $\mathbb{J}$ \\
\hline $\mathcal{H}_{3}\left(\mathbb{R}_{\mathbb{C}}\right) \simeq \operatorname{Sym}_{3}(\mathbb{C})$ & $\begin{array}{l}\text { 6-dimensional Lagrangian } \\
\text { grassmannian } \mathrm{LG}_{3}\left(\mathbb{C}^{6}\right) \subset \mathbb{P}^{13}\end{array}$ \\
\hline $\mathcal{H}_{3}\left(\mathbb{C}_{\mathbb{C}}\right) \simeq M_{3}(\mathbb{C})$ & $\begin{array}{l}\text { 9-dimensional Grassmannian } \\
\text { manifold } \mathrm{G}_{3}\left(\mathbb{C}^{6}\right) \subset \mathbb{P}^{19}\end{array}$ \\
\hline $\mathcal{H}_{3}\left(\mathbb{U}_{\mathbb{C}}\right) \simeq \mathrm{Alt}_{6}(\mathbb{C})$ & $\left.\begin{array}{l}\text { 15-dimensional orthogonal } \\
\text { Grassmannian OG }\end{array} \mathbb{C}^{12}\right) \subset \mathbb{P}^{31}$ \\
\hline $\mathcal{H}_{3}\left(\mathbb{O}_{\mathbb{C}}\right)$ & 27-dimensional $E_{7}$-variety in $\mathbb{P}^{55}$ \\
\hline
\end{tabular}


It follows from [39], [18] that the cubic curves $X_{\mathbb{J}}^{3}$ associated to one of the simple Jordan algebras presented in Table 1 are homogeneous varieties, yielding non-singular examples of $\bar{X}(k, 3,3)$ of Jordan type, for $k=6,9,15$ and 27.

The four varieties of the last column in Table 1 have been studied by several authors from many points of view. The interested reader can consult for example [46], [38], [39], [41], [18].

4.3.3. A Jordan cubic curve associated to the sextonions. The algebra of (complex) sextonions $\mathbb{S}_{\mathbb{C}}$ is an alternative algebra over $\mathbb{C}$ such that $\mathbb{U}_{\mathbb{C}} \subset \mathbb{S}_{\mathbb{C}} \subset \mathbb{O}_{\mathbb{C}}$. It is of (complex) dimension 6 and has been constructed in [40], [67].

The product (4.7) realizes $\mathcal{H}_{3}\left(\mathbb{S}_{\mathbb{C}}\right)$ as a 21-dimensional sub-Jordan algebra of $\mathcal{H}_{3}\left(\mathbb{O}_{\mathbb{C}}\right)$. Then $\mathcal{H}_{3}\left(\mathbb{S}_{\mathbb{C}}\right)$ is of rank 3 but is not semi-simple (cf. [40], Section 8.2). The cubic curve over $\mathcal{H}_{3}\left(\mathbb{S}_{\mathbb{C}}\right)$ is denoted by $G_{\omega}\left(\mathbb{\$}^{3}, \mathbb{S}^{6}\right)$ in [40] where it is explained why it can be considered as a kind of Lagrangian Grassmannian. It is a $\bar{X}(21,3,3)$ in $\mathbb{P}^{43}$ that is quasi-homogeneous and singular along a quadric of dimension 10 (cf. [40], Corollary 8.14).

4.4. Some cubic curves associated to associative algebras. Let $A$ be an associative algebra (of finite dimension) not necessarily commutative but with a unit $e$. Let $A^{+}$ denote the algebra $A$ endowed with the product $x * y=\frac{1}{2}(x y+y x)$. If $A$ is commutative, then $A=A^{+}$. It is immediate to see that in general $A^{+}$is a Jordan algebra with $e$ as unit. We will say that a Jordan algebra is special if it is isomorphic to a subalgebra of a Jordan algebra of the form $A^{+}$with $A$ associative. For instance, the simple Jordan algebras $\mathcal{H}_{3}\left(\mathbb{A}_{\mathbb{C}}\right)$ in Table 1 are special except when $\mathbb{A}=\mathbb{O}$.

Being associative, $A$ is also power-associative so that one can define its rank as introduced at the beginning of Section 4.3. Of course, the rank of the associative algebra $A$ and the rank of the associated Jordan algebra $A^{+}$coincide.

Complex associative algebras have been classified in low dimensions - e.g. in dimension 3, 4 and 5 - in classical or more recent papers and looking at these lists one immediately computes the rank of these algebras. Then, considering the $A^{+}$'s associated to rank three associative $A$ 's, one obtains examples of special cubic Jordan algebras in dimension 3,4 and 5. The computations needed to obtain the examples appearing in the following subsections are elementary but tedious and quite long so that they will not be reproduced here.

\subsubsection{The $\bar{X}(3,3,3)$ 's associated to special Jordan algebras of dimension three.} The classification of 3-dimensional complex associative algebras is classical, see [62], [57]. We refer to [23], [26] for more recent references.

Theorem 4.12. A 3-dimensional complex associative (unital) algebra of rank 3 is isomorphic to one of the following ones:

$$
A_{1}=\mathbb{C} \times \mathbb{C} \times \mathbb{C}, \quad A_{2}=\mathbb{C} \times \frac{\mathbb{C}[X]}{\left(X^{2}\right)}, \quad A_{3}=\frac{\mathbb{C}[X]}{\left(X^{3}\right)} .
$$


The above algebras are commutative so that $A_{i}^{+}=A_{i}$ (for $i=1,2,3$ ) are examples of special 3-dimensional cubic Jordan algebras. Let $X_{A_{i}}$ be the cubic curve associated to $A_{i}^{+}$. Clearly, the cubic curve $X_{A_{1}}$ is nothing but Segre's threefold $\mathbb{P}^{1} \times$ $\mathbb{P}^{1} \times \mathbb{P}^{1} \subset \mathbb{P}^{7}$, which is a particular case of the general construction of Lemma 4.11. Similarly, one verifies that $X_{A_{2}}$ is isomorphic to the Segre embedding of $\mathbb{P}^{1} \times S_{02}$ in $\mathbb{P}^{7}$.

On the other hand the cubic curve associated to $A_{3}$ yields a new example. One takes $x=1, y=X$ and $z=X^{2}$ as a $\mathbb{C}$-basis of $A_{3}$. Since this algebra is commutative, the Jordan product coincides with the associative one of $A_{3}$ and may be expressed as follows:

$$
(x, y, z) \cdot\left(x^{\prime}, y^{\prime}, z^{\prime}\right)=\left(x x^{\prime}, x y^{\prime}+x^{\prime} y, x z^{\prime}+x^{\prime} z+y y^{\prime}\right) .
$$

Then the generic norm and the adjoint of $(x, y, z) \in A_{3}$ are given by the following formulae:

$$
N(x, y, z)=x^{3} \quad \text { and } \quad(x, y, z)^{\#}=\left(x^{2},-x y, y^{2}-x z\right) .
$$

So the cubic curve $X_{A_{3}}$ is the closure of the image of the rational map

$$
[x: y: z: t] \longmapsto\left[t^{3}: x t^{2}: y t^{2}: z t^{2}: x^{2} t:-x y t:\left(y^{2}-x z\right) t: x^{3}\right] .
$$

The variety $X_{A_{3}} \subset \mathbb{P}^{7}$ can also be described as the image of $\mathbb{P}^{3}$ by the rational map associated to the linear system of cubic surfaces passing through three infinitely near double points (two of them generate the line $x=t=0$ contained in the base locus scheme of the linear system) so that it has degree 6.

4.4.2. Some $\bar{X}(4,3,3)$ associated to Jordan algebras of dimension four. The classification of 4-dimensional complex associative algebras is also classical, see [62], [58], and was also reconsidered more recently in [26], see also the references in these papers. As explained above, we are essentially interested in those of rank 3 , whose classification is contained in the next result where we use the labels and notation of [26], p. 151-152.

Proposition 4.13. Let $A$ be a 4-dimensional associative algebra of rank three. Then if $A$ is commutative, it is isomorphic to one of the following algebras

$$
A_{6}=\mathbb{C} \times \frac{\mathbb{C}[X, Y]}{(X, Y)^{2}}, \quad A_{7}=\frac{\mathbb{C}[X, Y]}{\left(X^{2}, Y^{2}\right)}, \quad A_{8}=\frac{\mathbb{C}[X, Y]}{\left(X^{3}, X Y, Y^{2}\right)}
$$

If $A$ is not commutative, then one of the following holds: 
- A is isomorphic to one of the following three triangular matrix algebras

$$
\begin{aligned}
A_{13}=\mathbb{C} \times\left(\begin{array}{ll}
\mathbb{C} & \mathbb{C} \\
0 & \mathbb{C}
\end{array}\right) ; \quad A_{14} & =\left\{\left(\begin{array}{lll}
a & 0 & 0 \\
c & a & 0 \\
d & 0 & b
\end{array}\right) \mid a, b, c, d \in \mathbb{C}\right\} ; \\
A_{15} & =\left(A_{14}\right)^{\mathrm{opp}} ;
\end{aligned}
$$

- there exists $\lambda \in \mathbb{C} \backslash\{1\}$ such that $A$ is isomorphic to

$$
A_{18(\lambda)}=\frac{\mathbb{C}\langle X, Y\rangle}{\left(X^{2}, Y^{2}, Y X-\lambda X Y\right)} ;
$$

- $A$ is isomorphic to

$$
A_{19}=\frac{\mathbb{C}\langle X, Y\rangle}{\left(Y^{2}, X^{2}+Y X, X Y+Y X\right)} .
$$

(Here $\mathbb{C}\langle X, Y\rangle$ stands for the free associative algebra generated by $1, X$ and $Y$ ).

Since $A_{15}=\left(A_{14}\right)^{\text {opp }}$ one has $A_{14}^{+}=A_{15}^{+}$. Moreover, one verifies easily (via elementary computations) that the Jordan algebras $A_{18(\lambda)}^{+}$and $A_{19}^{+}$are associative so that they are isomorphic to one of the three commutative associative algebras $A_{6}, A_{7}$ or $A_{8}$ (in fact one has $A_{18(\lambda)}^{+} \simeq A_{7}$ and $A_{19}^{+} \simeq A_{8}$ ). Thus we get the following result.

Corollary 4.14. Let $\mathbb{J}$ be a rank 3 Jordan algebra of dimension 4 of the form $A^{+}$ with $A$ associative and of rank three. Then $\mathbb{J}$ is isomorphic to one of the following algebras:

$$
A_{6}, \quad A_{7}, \quad A_{8}, \quad A_{13}^{+} \text {or } A_{14}^{+} .
$$

The reader has to be aware that not every 4-dimensional cubic Jordan algebra is of the form $A^{+}$with $A$ associative. For instance (as explained above), if $\mathbb{J}^{\prime}$ stands for the simple Jordan algebra of rank 2 on $\mathbb{C}^{3}$ then the direct product $\mathbb{C} \times \mathbb{J}^{\prime}$ is a non-associative Jordan cubic algebra not isomorphic to any of the Jordan algebras in (4.9). In this case, the associated cubic curve $X_{\mathbb{C} \times \mathbb{J}^{\prime}}$ is $\mathbb{P}^{1} \times Q \subset \mathbb{P}^{9}$ where $Q$ is a smooth hyperquadric in $\mathbb{P}^{4}$.

Another example is given by the Jordan algebra denoted by $\mathbb{J}_{*}$, the Jordan product of which is explicitly given by $x * y=\left(-x_{1} y_{1}, x_{2} y_{2}, x_{4} y_{4}-x_{1} y_{3}-x_{3} y_{1}, \frac{1}{2}\left(x_{2} y_{4}+x_{4} y_{2}-x_{1} y_{4}-x_{4} y_{1}\right)\right)$ for $x=\left(x_{i}\right)_{i=1}^{4}$ and $y=\left(y_{i}\right)_{i=1}^{4}$ in $\mathbb{C}^{4}=\mathbb{J}_{*}$. One verifies that $\mathbb{J}_{*}$ is indeed of rank 3 . 
After some easy computations, one obtains explicit affine parametrizations of the form $x \mapsto\left[1: x: x^{\#}: N(x)\right]$ of the cubic curves associated to the cubic Jordan algebras mentioned above in this subsection. We collect them in the following table for further reference.

Table 2

\begin{tabular}{|l|l|c|c|}
\hline Algebra $\mathbb{J}$ & \multicolumn{1}{|c|}{ Adjoint $\boldsymbol{x}^{\#}$} & Norm $N(\boldsymbol{x})$ & $\boldsymbol{X}_{\mathbb{J}} \subset \mathbb{P}^{\mathbf{9}}$ \\
\hline$A_{6}^{+}=A_{6}$ & $\left(x_{2}{ }^{2}, x_{1} x_{2},-x_{1} x_{3},-x_{1} x_{4}\right)$ & $x_{1} x_{2}{ }^{2}$ & $\mathbb{P}^{1} \times S_{002}$ \\
\hline$A_{7}^{+}=A_{7}$ & $\left(x_{1}{ }^{2},-x_{1} x_{2},-x_{1} x_{3}, 2 x_{2} x_{3}-x_{1} x_{4}\right)$ & $x_{1}{ }^{3}$ & \\
\hline$A_{8}^{+}=A_{8}$ & $\left(x_{1}{ }^{2},-x_{1} x_{2},-x_{1} x_{3}, x_{2}{ }^{2}-x_{1} x_{4}\right)$ & $x_{1}{ }^{3}$ & \\
\hline$A_{13}^{+}$ & $\left(x_{2} x_{4}, x_{1} x_{4},-x_{1} x_{3}, x_{1} x_{2}\right)$ & $x_{1} x_{2} x_{4}$ & $\mathbb{P}^{1} \times S_{011}$ \\
\hline$A_{14}^{+}$ & $\left(x_{1} x_{2}, x_{1}{ }^{2},-x_{2} x_{3},-x_{1} x_{4}\right)$ & $x_{1}{ }^{2} x_{2}$ & \\
\hline $\mathbb{C} \times \mathbb{J}^{\prime}$ & $\left(x_{2}^{2}+x_{3}^{2}+x_{4}^{2}, x_{1} x_{2},-x_{1} x_{3},-x_{1} x_{4}\right)$ & $x_{1}\left(x_{2}^{2}+x_{3}^{2}+x_{4}^{2}\right)$ & $\mathbb{P}^{1} \times Q$ \\
\hline $\mathbb{J}_{*}$ & $\left(x_{1} x_{2}, x_{1}{ }^{2}, x_{4}{ }^{2}-x_{2} x_{3}, x_{1} x_{4}\right)$ & $x_{1}{ }^{2} x_{2}$ & \\
\hline
\end{tabular}

4.5. Some other examples of $\bar{X}(r+1, n, 2 n-3)$ when $n>3$. According to the main result of [52], a variety $\bar{X}(r+1, n, \delta)$ is of Castelnuovo type except maybe when $n>2, r>1$ and $\delta=2 n-3$. The cubic curves associated to Jordan algebras of rank three provide examples of $\bar{X}(r+1,3,3)$ that are not of Castelnuovo type. It is natural to try to produce some examples of $\bar{X}(r+1, n, 2 n-3)$ not of Castelnuovo type for $n>3$.

We are aware essentially only of examples which are closely related to varieties 3 -covered by twisted cubics.

The Veronese manifold $v_{3}\left(\mathbb{P}^{3}\right) \subset \mathbb{P}^{19}$ is of course a $\bar{X}(3,2,3)$ but is also a $\bar{X}(3,6,9)$. Indeed, since $\mathbb{P}^{3}$ is 6-covered by twisted cubics, it follows that $v_{3}\left(\mathbb{P}^{3}\right)$ is 6-covered by rational normal curves of degree 9. Of course, $v_{3}\left(\mathbb{P}^{3}\right)$ is not of Castelnuovo type (since $\operatorname{deg}\left(v_{3}\left(\mathbb{P}^{3}\right)\right)=27$ whereas the degree of a Castelnuovo model $X=\bar{X}(3,6,9)$ is 17$)$. Now let $x_{1}, x_{2}, x_{3}$ be three generic points on $v_{3}\left(\mathbb{P}^{3}\right)$. For $I \subset\{1,2,3\}$ of cardinality $i \leq 3$, let $X_{I}$ be the image of $v_{3}\left(\mathbb{P}^{3}\right)$ by the osculating projection of center $S_{I}$ defined as the span of the 1-osculating spaces of $v_{3}\left(\mathbb{P}^{3}\right)$ at the points $x_{i}$ with $i \in I$. Then $X_{I}$ is non-degenerate in $\mathbb{P}^{19-4 i}$, is $(6-i)$-covered by rational normal curves of degree $9-2 i$ hence is an example of $\bar{X}(3,6-i, 9-2 i)$. When $i=3$, one has $X_{I}=\mathbb{P}^{1} \times \mathbb{P}^{1} \times \mathbb{P}^{1}$ hence this example is not new. But for $i=1$ and $i=2$, one obtains respectively two new examples of $\bar{X}(3,5,7)$ and $\bar{X}(3,4,5)$ that are not of Castelnuovo type. 


\section{Classification of projective varieties 3-covered by twisted cubics}

By definition $\bar{\pi}(r, 3,3)=2 r+4$ for every $r \geq 1$. In this Section we shall classify varieties $X=\bar{X}(r+1,3,3) \subset \mathbb{P}^{2 r+3}$ for $r$ small and/or under suitable hypothesis.

Let us recall some facts, which were proved in the previous sections or which are easy consequences of them.

Lemma 5.1. Let $X=\bar{X}(r+1,3,3) \subset \mathbb{P}^{2 r+3}$. Then:

(1) The tangential projection $\pi_{T}: X \rightarrow \mathbb{P}^{r+1}$ from the tangent space $T=T_{x} X=$ $\operatorname{Osc}_{X}^{1}(x)$ at a general point $x \in X$ is birational. In particular $X$ is a rational variety, $S X=\mathbb{P}^{2 r+3}$ and $X$ is not a cone.

(2) The variety $X \subset \mathbb{P}^{2 r+3}$ is not the birational projection from an external point of a variety $X^{\prime} \subset \mathbb{P}^{2 r+4}$.

Proof. The family of twisted cubics passing through $x$ is 2-covering and a general twisted cubic in this family projects from $T$ onto a general line in $\mathbb{P}^{r+1}$ so that the birationality of $\pi_{T}$ follows from Lemma 2.1 and the first part is proved.

Suppose that $X=\pi_{p}\left(X^{\prime}\right)$ with $p \in \mathbb{P}^{2 r+4} \backslash X^{\prime}$. Let $x_{i} \in X, i=1,2,3$, be general points and let $x_{i}^{\prime} \in X^{\prime}$ such that $\pi_{p}\left(x_{i}^{\prime}\right)=x_{i}$ for every $i=1,2,3$. If $C \subset X$ is the unique twisted cubic passing through $x_{1}, x_{2}, x_{3}$ and if $C^{\prime} \subset X^{\prime}$ is its strict transform on $X^{\prime}$, then $C^{\prime}$ is a rational curve passing through $x_{1}^{\prime}, x_{2}^{\prime}$, $x_{3}^{\prime}$ such that $\pi_{p}\left(C^{\prime}\right)=C$, yielding $\operatorname{deg}\left(C^{\prime}\right)=\operatorname{deg}(C)=3$. This would imply $X^{\prime}=X(r+1,3,3) \subset \mathbb{P}^{2 r+4}$, and we would obtain $2 r+5 \leq \bar{\pi}(r, 3,3)=2 r+4$. This contradiction concludes the proof.

By definition, there exists an irreducible family of twisted cubics, let us say $\Sigma$, contained in $X=\bar{X}(r+1,3,3) \subset \mathbb{P}^{2 r+3}$. Moreover, $\Sigma$ has dimension $3 r$, is 3covering and through three general points of $X$ there passes a unique twisted cubic belonging to $\Sigma$. The family of twisted cubics in $\Sigma$ passing through a general point $x \in X$ contains an irreducible component of dimension $2 r$ which is 2-covering for $X$. These twisted cubics are mapped by $\pi_{T}$ onto the lines in $\mathbb{P}^{r+1}$ and a general line in $\mathbb{P}^{r+1}$ is the image via $\pi_{T}$ of a twisted cubic passing through $x$. The birational map

$$
\phi=\pi_{T}^{-1}: \mathbb{P}^{r+1} \rightarrow X \subset \mathbb{P}^{2 r+3}
$$

is thus given by a linear system of cubic hypersurfaces mapping a general line of $\mathrm{P}^{r+1}$ birationally onto a twisted cubic passing through $x$. The general cubic hypersurface in this linear system is mapped by $\phi$ birationally onto a general hyperplane section of $X$.

Let $\bar{\pi}_{x}: \bar{X}=\mathrm{Bl}_{x}(X) \rightarrow X$ be the blow-up of $X$ at $x$ and let $E=\mathbb{P}^{r}$ be the exceptional divisor of $\bar{\pi}_{x}$. Let $\bar{\pi}_{T}=\bar{\pi}_{x} \circ \pi_{T}: \bar{X} \rightarrow \mathbb{P}^{r+1}$. The restriction of $\bar{\pi}_{T}$ to $E$ is defined by a linear system $\left|I I_{X, x}\right|$ of quadric hypersurfaces in $E=\mathbb{P}^{r}$, the 
so-called second fundamental form of $X$ at $x$. We shall denote by $B_{x} \subset E=\mathbb{P}^{r}$ the base locus scheme of $\left|I I_{X, x}\right|$.

Since $X \subset \mathbb{P}^{2 r+3}$ is non-degenerate, the birational map $\bar{\pi}_{T}$ is defined at the general point of $E$. We claim that $E^{\prime}=\bar{\pi}_{T \mid E}(E)=\mathbb{P}^{r} \subset \mathbb{P}^{r+1}$ is a hyperplane and that the restriction of $\bar{\pi}_{T}$ to $E$ is birational onto its image. Indeed, if $\operatorname{dim}\left(E^{\prime}\right)<r$, then a general line in $\mathbb{P}^{r+1}$ would not cut $E^{\prime}$ and its image by $\phi$ would not pass through $x$. If $\operatorname{deg}\left(E^{\prime}\right) \geq 2$, then a general line $l \subset \mathbb{P}^{r+1}$ would cut $E^{\prime}$ at $\operatorname{deg}\left(E^{\prime}\right)$ distinct points where $\phi$ is defined. From $\phi\left(E^{\prime}\right)=x$ we would deduce that $\phi(l)$ is singular at $x$, in contrast with the fact that $\phi(l)$ is a twisted cubic. From this picture it also immediately follows the birationality of the restriction of $\bar{\pi}_{T}$ to $E$.

Therefore $\operatorname{dim}\left(\left|I I_{X, x}\right|\right)=r$ and $\bar{\pi}_{T \mid E}: E \rightarrow E^{\prime}$ is a Cremona transformation not defined along $B_{x}$, the base locus scheme of $\left|I I_{X, x}\right|$. Moreover, since $\phi\left(E^{\prime}\right)=x$, the restriction of the linear system of cubic hypersurfaces defining $\phi$ to $E^{\prime}$ is constant and given by a cubic hypersurface $\mathrm{C}_{x}^{\prime} \subset E^{\prime}=\mathbb{P}^{r}$. One can assume that $E^{\prime} \subset \mathbb{P}^{r+1}$ is cut out by $x_{0}=0$. Let $\boldsymbol{x}=\left(x_{1}, \ldots, x_{r+1}\right) \in \mathbb{C}^{r+1}$ and let $f(\boldsymbol{x})$ be a cubic equation for $\mathrm{C}_{x}^{\prime} \subset E^{\prime}$. Let us choose homogeneous coordinates $\left(y_{0}: \cdots: y_{2} r+3\right)$ on $\mathbb{P}^{2 r+3}$ such that $x=(0: \cdots: 0: 1)$ and $T_{x} X=V\left(y_{0}, \ldots, y_{r+1}\right)$.

The map $\phi: \mathbb{P}^{r+1} \rightarrow X \subset \mathbb{P}^{2 r+3}$ is given by $2 r+4$ cubic polynomials $g_{0}, \ldots, g_{2 r+3}$. We can suppose that $x_{0}$ does not divide $g_{2 r+3}$ and that $x_{0}$ divides $g_{j}$ for every $j=0, \ldots, 2 r+2$. Moreover $x_{0}^{2}$ divides $g_{0}, \ldots g_{r+1}$ since the hyperplane sections of the form $\lambda_{0} g_{0}+\cdots+\lambda_{r+1} g_{r+1}=0$ correspond to hyperplane sections of $X$ containing $T_{x} X$ and hence having at least a double point at $x$. Modulo a change of coordinates on $\mathbb{P}^{r+1}$ we can thus suppose $g_{i}=x_{0}^{2} x_{i}$ for every $i=0, \ldots, r+1$ and that $g_{2 r+3}=x_{0} g+f$, with $g=g(x)$ quadratic polynomial. The hyperplane sections of $X$ passing through $x$ and not containing $T_{x} X$ are smooth at $x$ from which it follows that we can also suppose $g_{r+2+j}=x_{0} f_{j}$ with $j=0, \ldots, r$ and $f_{j}=f_{j}(\boldsymbol{x})$ quadratic polynomials. By Lemma 5.1 we can also suppose $g=0$, or equivalently $g \in\left\langle f_{0}, \ldots, f_{r}\right\rangle$. Otherwise $X \subset \mathbb{P}^{2 r+3}$ would be the birational projection on the hyperplane $V\left(y_{2 r+4}\right)=\mathbb{P}^{2 r+3} \subset \mathbb{P}^{2 r+4}$ from the external point $(0: \cdots: 0: 1:-1) \in \mathbb{P}^{2 r+4}$ of the variety $X^{\prime} \subset \mathbb{P}^{2 r+4}$ having the parametrization $\tilde{\phi}: \mathbb{P}^{r+1} \rightarrow X^{\prime} \subset \mathbb{P}^{2 r+4}$ given by the following homogenous cubic polynomials: $\tilde{g}_{i}=g_{i}$ for $i=0, \ldots, 2 r+2 ; \tilde{g}_{2 r+3}=x_{0} g$ and $\tilde{g}_{2 r+4}=f$.

By blowing-up the point $x$ on $\mathbb{P}^{2 r+3}$ it immediately follows that the restriction of $\bar{\pi}_{T}^{-1}$ to $E^{\prime}$ is given by $\left(f_{0}: \cdots: f_{r}\right)$. Hence $\psi_{x}:=\bar{\pi}_{T \mid E}: E \rightarrow E^{\prime}$ is either a projective transformation or a Cremona transformation of type $(2,2)$, i.e. given by quadratic forms without a common factor and such that the inverse is also given by quadratic forms without a common factor. In conclusion we can suppose that the rational map $\phi$ is given by the $2 r+4$ cubic polynomials

$$
x_{0}^{3}, x_{0}^{2} x_{1}, \ldots, x_{0}^{2} x_{r+1}, x_{0} f_{0}, \ldots, x_{0} f_{r}, f
$$

and that the base locus of $\psi_{x}^{-1}, B_{x}^{\prime} \subset \mathbb{P}^{r}=E^{\prime}$, is $V\left(f_{0}, \ldots, f_{r}\right) \subset \mathbb{P}^{r}$, where 
in this case the polynomials $f_{i}(\boldsymbol{x})$ are considered as polynomials in the variables $x_{1}, \ldots, x_{r+1}$.

Let $t_{x} X$ denote the affine tangent space to $X$ at $x$. The first principal result in this section is the following.

Theorem 5.2. Let $X=\bar{X}(r+1,3,3) \subset \mathbb{P}^{2 r+3}$ and let notation be as above. Let $x \in X$ be general and let $\psi_{x}: \mathbb{P}^{r} \rightarrow \mathbb{P}^{r}$ be the associated Cremona transformation. Then the following conditions are equivalent:

(a) $\psi_{x}$ is equivalent to a projective transformation as a birational map;

(b) $X$ is projectively equivalent either to $S_{1 \ldots 122}$ or to $S_{1 \ldots 113}$;

(c) the affine parametrization deduced from (5.1) is, respectively, either

$$
\left(1: x_{1}: \cdots: x_{r+1}: x_{1}^{2}: x_{1} x_{2}: \cdots: x_{1} x_{r+1}: x_{1}^{2} x_{2}\right)
$$

or

$$
\left(1: x_{1}: \cdots: x_{r+1}: x_{1}^{2}, x_{1} x_{2}: \cdots: x_{1} x_{r+1}: x_{1}^{3}\right) ;
$$

(d) the projection from $T=T_{x} X$ of a general twisted cubic included in $X$ is a conic.

If $X=\bar{X}(r+1,3,3) \subset \mathbb{P}^{2 r+3}$ is not a rational normal scroll as above, then:

(1) the associated Cremona transformation $\psi_{x}$ is of type $(2,2)$;

(2) the linear system defining $\phi: \mathbb{P}^{r+1} \rightarrow X \subset \mathbb{P}^{2 r+3}$ consists of the cubic hypersurfaces in $\mathbb{P}^{r+1}$ having double points along $B_{x}^{\prime} \subset E^{\prime} \subset \mathbb{P}^{r+1}$;

(3) the scheme $B_{x} \subset \mathbb{P}^{r}$ is equal (as scheme) to $\mathscr{L}_{x} \subset \mathbb{P}^{r}$, the Hilbert scheme of lines contained in $X$ and passing through $x$ in its natural embedding into $E=\mathbb{P}\left(\left(t_{x} X\right)^{*}\right)$. Moreover, $B_{x}^{\prime} \subset E^{\prime}=\mathbb{P}^{r}$ and $B_{x}=\mathscr{L}_{x} \subset E=\mathbb{P}^{r}$ are projectively equivalent so that $\psi_{x}$ and its inverse have the same base loci, modulo this identification.

(4) if $X$ is also smooth, then $B_{x}=\mathscr{L}_{x}$ and $B_{x}^{\prime}$ are smooth schemes.

Proof. The birational map $\psi_{x}$ is equivalent to a projective transformation as a birational map if and only if the linear system $\left|I I_{X, x}\right|$ has a fixed component, which is necessarily a hyperplane. It is well known, see also [29], (3.21), that this happens if and only if $X \subset \mathbb{P}^{2 r+3}$ is a scroll in the classical sense. From Lemma 5.1 we deduce that this happens if and only if $X \subset \mathbb{P}^{2 r+3}$ is a smooth rational normal scroll, yielding the equivalence of (a) and (b). If $X \subset \mathbb{P}^{2 r+3}$ is a rational normal scroll as before, a general twisted cubic $\bar{C} \subset X$ cuts $\mathbb{P}_{x}^{r}=T_{x} X \cap X$ in a point so that $\pi_{T}(\bar{C})=\widetilde{C} \subset \mathbb{P}^{r+1}$ is a conic cutting $E^{\prime}$ in two points, possibly coincident. These two points are contained in the base locus of $\psi$ and one of this point is double for the general cubic hypersurface in the linear system defining $\psi$ since $\psi(\widetilde{C})=\bar{C}$ is a 
twisted cubic. From this we deduce that $\mathcal{C}_{x}^{\prime}$ consists of a double hyperplane $\Pi \subset E^{\prime}$, which is exactly $B_{x}^{\prime}$, and of another hyperplane, possibly infinitely near to $\Pi$. By reversing the construction we see that if $\pi_{T}(\bar{C})=\widetilde{C}$ is a conic, then $\mathrm{C}_{x}^{\prime}$ is as before and the general cubic hypersurface defining $\phi$ has a double point along a hyperplane $\Pi \subset E^{\prime}$, which is easily seen to be equal to $B_{x}^{\prime}$, yielding that $X \subset \mathbb{P}^{2 r+3}$ is a scroll. Therefore also the equivalence of (b) and (d) is proved. If we suppose that $B_{x}^{\prime}=\mathbb{P}^{r-1} \subset E^{\prime}$ is given by $x_{0}=x_{1}=0$ and if we take into account the previous description we immediately deduce the equivalence between (c) and (d).

Suppose from now on that $X=\bar{X}(r+1,3,3) \subset \mathbb{P}^{2 r+3}$ is not a rational normal scroll so that by the previous part the associated Cremona map $\psi_{x}$ is of type $(2,2)$, proving (1).

By the discussion above on $\mathbb{P}^{r+1} \backslash E^{\prime}$ the map $\phi$ has an affine expression

$$
\phi(\boldsymbol{x})=\left(1: x_{1}: \cdots: x_{r+1}: f_{0}(\boldsymbol{x}): \cdots: f_{r}(\boldsymbol{x}): f(\boldsymbol{x})\right) .
$$

Let $\left(y_{0}: \cdots: y_{2 r+3}\right)$ be a system of homogeneous coordinates on $\mathbb{P}^{2 r+3}$ as above. Then the equations defining $X \subset \mathbb{P}^{2 r+3}$ in the affine space $\mathbb{A}^{2 r+3}=\mathbb{P}^{2 r+3} \backslash V\left(y_{0}\right)$ are $y_{i}=x_{i}, i=1, \ldots, r+1 ; y_{r+2+j}=f_{j}(\boldsymbol{x}), j=0, \ldots, r$ and $y_{2 r+3}=f(\boldsymbol{x})$, that is, letting $\boldsymbol{y}=\left(y_{1}, \ldots, y_{r+1}\right)$, we get the equations $y_{r+2+j}=f_{j}(\boldsymbol{y})$ for $j=0, \ldots, r$ and $y_{2 r+3}=f(\boldsymbol{y})$.

Let $p=\phi(1: \boldsymbol{p})=\left(1: \boldsymbol{p}: f_{0}(\boldsymbol{p}): \cdots: f_{r}(\boldsymbol{p}): f(\boldsymbol{p})\right)$ be a general point of $X$, with $\boldsymbol{p}=\left(p_{1}, \ldots, p_{r+1}\right) \in \mathbb{C}^{r+1}$. In particular $(0: \boldsymbol{p})$ is a general point on $E^{\prime}$. A tangent direction at $p \in X$ corresponds to the image via $d \phi_{q}$ of the tangent direction to some line passing through $q=(1: p) \in \mathbb{P}^{r+1}$. We shall parametrize lines through $q$ via points $(0: \boldsymbol{y}) \in E^{\prime}$ so that such a line, denoted by $L_{y}$, admits $t \mapsto \boldsymbol{p}+t \boldsymbol{y}$ as an affine parametrization. Then for $i=0, \ldots, r$, one has

$$
f_{i}(\boldsymbol{p}+t \boldsymbol{y})=f_{i}(\boldsymbol{p})+2 t f_{i}^{1}(\boldsymbol{p}, \boldsymbol{y})+t^{2} f_{i}(\boldsymbol{y}),
$$

where $f_{i}{ }^{1}$ stands for the bilinear form associated to the quadratic form $f_{i}$. Moreover

$$
f(\boldsymbol{p}+t \boldsymbol{y})=f(\boldsymbol{p})+t f(\boldsymbol{p}, \boldsymbol{y})+t^{2} f(\boldsymbol{y}, \boldsymbol{p})+t^{3} f(\boldsymbol{y}),
$$

where $f(\boldsymbol{p}, \boldsymbol{y})=d f_{\boldsymbol{p}}(\boldsymbol{y})$ is quadratic in $\boldsymbol{p}$ and linear in $\boldsymbol{y}$.

Clearly, the base locus of the second fundamental form at $p=\phi(1: p)$ is the scheme

$$
B_{p}=V\left(f_{0}(\boldsymbol{y}), \ldots, f_{r}(\boldsymbol{y}), f(\boldsymbol{y}, \boldsymbol{p})\right)=V\left(f_{0}(\boldsymbol{y}), \ldots, f_{r}(\boldsymbol{y})\right) \subset \mathbb{P}\left(\left(t_{p} X\right)^{*}\right),
$$

where the second equality of schemes follows from the equality $\operatorname{dim}\left(\left\langle f_{0}, \ldots, f_{r}\right\rangle\right)=$ $r+1$ combined with the fact that $\operatorname{dim}\left(\left|I I_{X, p}\right|\right)=\operatorname{dim}\left(\left|I I_{X, x}\right|\right)$ by the generality of $p \in X$. In particular we deduce that for $z \in B_{p}$ we have $f(\boldsymbol{z}, \boldsymbol{p})=0$. Because $(0: \boldsymbol{p})$ is general in $E^{\prime}$, this implies $d f_{z}=0$ (since $f(\boldsymbol{z}, \boldsymbol{p})=d f_{\boldsymbol{z}}(\boldsymbol{p})$ for every $\boldsymbol{p}$ ) on one hand, and gives $f(z)=0$ on the other hand (since $0=f(z, z)=3 f(z)$ after 
specializing $\boldsymbol{p}=\boldsymbol{z})$. The previous facts show that the cubic $\mathrm{C}_{x}^{\prime}=V(f(\boldsymbol{x})) \subset \mathbb{P}^{r+1}$ has double points along $B_{x}^{\prime}$ and part (2) is proved. From these facts it immediately follows also that the closure of the image of the line $L_{z}$ (for $z \in B_{p}$ ) via the map $\phi$ is a line included in $X$ and passing through $p$, proving (3). Put more intrinsically, the equation of $\mathscr{L}_{p}$, the Hilbert scheme of lines contained in $X$ and passing through $p$ in its natural embedding into $\mathbb{P}\left(\left(t_{p} X\right)^{*}\right)$, is the scheme defined by the equations $f_{j}(\boldsymbol{x}), f(\boldsymbol{x}, \boldsymbol{p}), f(\boldsymbol{x})$ and we proved that the ideal generated by these polynomials coincides with the ideal generated by the $f_{j}$ 's which defines $B_{p}$ as a scheme.

To prove (4) we recall that for a smooth variety $X \subset \mathbb{P}^{N}$ the scheme $\mathscr{L}_{x} \subset$ $\mathbb{P}\left(\left(t_{x} X\right)^{*}\right)$, when non-empty, is a smooth scheme for $x \in X$ general, see for example Proposition 2.2 of [53].

Let

$$
\varphi=\left(\varphi_{0}, \ldots, \varphi_{r}\right): \mathbb{P}^{r} \rightarrow \mathbb{P}^{r}
$$

be a Cremona transformation of bidegree $(2,2)$. Let $B$, respectively $B^{\prime}$, be the base locus of $\varphi$, respectively of $\varphi^{-1}$. The classification of such maps is known for $r=2$, $r=3$ (see [47]) and for $r=4$ in the generic case (see [60]). From this classification one deduces that in low dimension the base loci $B$ and $B^{\prime}$ are projectively equivalent so that, modulo a projective transformation, every Cremona transformation of bidegree $(2,2)$ in $\mathbb{P}^{r}, r \leq 4$, is an involution. As a consequence of Theorem 5.2 we deduce below that this holds for arbitrary $r \geq 2$ a priori and not as a consequence of the classification. As far we know, this question has not been addressed in the literature.

Corollary 5.3. Let $\varphi=\left(\varphi_{0}, \ldots, \varphi_{r}\right): \mathbb{P}^{r} \rightarrow \mathbb{P}^{r}$ be a Cremona transformation of bidegree $(2,2)$ with $r \geq 2$. Let $B$, respectively $B^{\prime}$, be the base locus of $\varphi$, respectively of $\varphi^{-1}$. Then $B$ and $B^{\prime}$ are projectively equivalent.

\section{Proof. Consider}

$$
\pi_{1}: \mathrm{Bl}_{B}\left(\mathbb{P}^{r}\right) \rightarrow \mathbb{P}^{r},
$$

the blow-up of $\mathbb{P}^{r}$ along $B$, and

$$
\pi_{2}: \mathrm{Bl}_{B^{\prime}}\left(\mathbb{P}^{r}\right) \rightarrow \mathbb{P}^{r},
$$

the blow-up of $\mathbb{P}^{r}$ along $B^{\prime}$. We deduce the following diagram of birational maps:

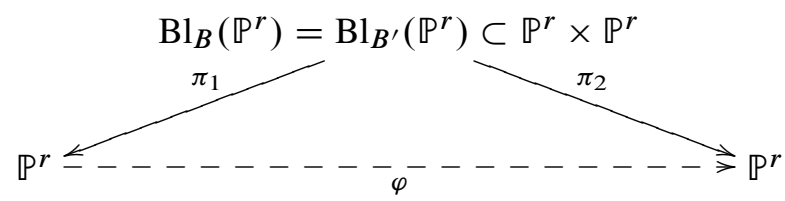

where $\pi_{i}$ are naturally identified with the restriction of the projections on each factor. The equality $\mathrm{Bl}_{B}\left(\mathbb{P}^{r}\right)=\mathrm{Bl}_{B^{\prime}}\left(\mathbb{P}^{r}\right)$, from now on indicated with $\widetilde{\mathbb{P}}^{r}$, follows from the 
fact that these reduced and irreducible schemes are the closure of the graph of the maps $\varphi$ and $\varphi^{-1}$ inside $\mathbb{P}^{r} \times \mathbb{P}^{r}$.

Let $E_{1}=\pi_{1}^{-1}(B)$ and $E_{2}=\pi_{2}^{-1}\left(B^{\prime}\right)$ be the $\pi_{i}$-exceptional Cartier divisors, $i=1,2$, defined by the invertible sheaves $\pi_{1}^{-1} \mathcal{I}_{B} \cdot \mathcal{O}_{\widetilde{\mathbb{P}} r}$, respectively $\pi_{2}^{-1} \mathcal{I}_{B^{\prime}} \cdot \mathcal{O}_{\widetilde{\mathbb{P}} r}$. Let $H_{i} \in\left|\pi_{i}^{*}(\mathcal{O}(1))\right|$ for $i=1,2$. We have

$$
H_{2} \sim 2 H_{1}-E_{1} \quad \text { and } \quad H_{1} \sim 2 H_{2}-E_{2}
$$

from which we deduce

$$
E_{1} \sim 3 H_{2}-2 E_{2} \text { and } E_{2} \sim 3 H_{1}-2 E_{1} .
$$

Let $\boldsymbol{x}=\left(x_{1}: \cdots: x_{r+1}\right)$ be homogeneous coordinates in $\mathbb{P}^{r}$, which we shall consider as the hyperplane $V\left(x_{0}\right)$ on $\mathbb{P}^{r+1}$ with homogeneous coordinates $\left(x_{0}: x_{1}\right.$ : $\left.\cdots: x_{r+1}\right)$. Let $C_{1}=\pi_{1}\left(E_{2}\right)=V(n(\boldsymbol{x})) \subset \mathbb{P}^{r}$ and let $C_{2}=\pi_{2}\left(E_{1}\right)=V(\tilde{n}(\boldsymbol{x}))$. By the above description $C_{1} \subset \mathbb{P}^{r}$ is a cubic hypersurface singular along $B$, that is the partial derivatives of $n(\boldsymbol{x})$ also belong to the homogeneous saturated ideal $I_{B} \subset \mathbb{C}\left[x_{1}, \ldots, x_{r+1}\right]$. One also immediately proves that $C_{1}$ is the so-called secant scheme of $B$, that is the scheme defined by the image of the universal family of lines in $\mathbb{P}^{2 r+3}$ over the lines generated by length 2 subschemes of $B$. From this one deduces another proof that $C_{1}$ is singular along $B$.

The map $\phi: \mathbb{P}^{r+1} \rightarrow \mathbb{P}^{2 r+3}$ given by

$$
\phi\left(x_{0}, \boldsymbol{x}\right)=\left(x_{0}^{3}: x_{0}^{2} x_{1}: \cdots: x_{0}^{2} x_{r+1}: x_{0} \varphi_{0}(\boldsymbol{x}): \cdots: x_{0} \varphi_{r}(\boldsymbol{x}): n(\boldsymbol{x})\right)
$$

is birational onto the closure of its image $X \subset \mathbb{P}^{2 r+3}$. We claim that $X=\bar{X}(r+$ $1,3,3)$ so that the conclusion will follow from part (3) of Theorem 5.2. Indeed let $p_{1}, p_{2}, p_{3} \in \mathbb{P}^{r+1}$ be three general points, let $\Pi=\left\langle p_{1}, p_{2}, p_{3}\right\rangle$ be the plane they span and let $L=V\left(x_{0}\right) \cap \Pi$. Then $L \subset V\left(x_{0}\right)=\mathbb{P}^{r}$ is a general line so that $\varphi(L)=C$ is a conic in $\mathbb{P}^{r}$ cutting $B^{\prime}$ in a length 3 scheme $R^{\prime}$ spanning a plane $\Pi^{\prime}$. Then $\varphi^{-1}\left(\Pi^{\prime}\right)=\Pi$ is a plane containing $L$ and cutting $B$ in a length 3 scheme $R$ spanning $\Pi$. Then $\left\langle p_{1}, p_{2}, p_{3}, R\right\rangle=\mathbb{P}^{3}$ and through the length 6 scheme $p_{1} \cup p_{2} \cup p_{3} \cup R$ there passes a unique twisted cubic $\widetilde{C}$. Then $\phi(\widetilde{C})$ is a twisted cubic since the linear system defining $\phi$ consists of cubic hypersurfaces having double points along $B$ and it passes through the three general points $\phi\left(p_{1}\right), \phi\left(p_{2}\right), \phi\left(p_{3}\right) \in X$.

Let the notation be as above. Then, modulo composition to the left by a linear map, one can assume that $B=B^{\prime}$. This implies in particular that there exist $\ell \in$ $\operatorname{End}\left(\mathbb{C}^{r+1}\right)$ invertible such that $\varphi^{-1}=\ell \circ \varphi$. Thus there exists a cubic form $n(\boldsymbol{x})$ such that

$$
\ell \circ \varphi \circ \varphi(\boldsymbol{x})=n(\boldsymbol{x}) \boldsymbol{x}
$$

for every $\boldsymbol{x}=\left(x_{1}, \ldots, x_{r+1}\right) \in \mathbb{C}^{r+1}$. 
In substance, Theorem 5.2 and the construction in Corollary 5.3 say that every $\bar{X}(r+1,3,3)$ not of Castelnuovo type determines (and is determined by) a quadroquadric Cremona transformation of $\mathbb{P}^{r}$. We point out that the previous remark has the following interesting geometric consequence.

Corollary 5.4. Let $X=\bar{X}(r+1,3,3) \subset \mathbb{P}^{2 r+3}$. Then $X$ is a variety with one apparent double point, that is there passes a unique secant line to $X$ through a general point $q \in \mathbb{P}^{2 r+3}$.

Proof. Let notation be as above and let $q=\left(1: \boldsymbol{q}: \boldsymbol{q}^{\prime}: z\right) \in \mathbb{P}^{2 r+3}$ be a fixed general point, where $\boldsymbol{q}, \boldsymbol{q}^{\prime} \in \mathbb{C}^{r+1}$ and $z \in \mathbb{C}^{*}$ by generality of $q$. Moreover, we can also suppose that all the pairs of distinct points $p_{1}, p_{2} \in X$ such that $q \in\left\langle p_{1}, p_{2}\right\rangle$ are of the form $p_{i}=\left(1: \boldsymbol{x}_{i}: \boldsymbol{x}_{i}^{\prime}: n\left(\boldsymbol{x}_{i}\right)\right)$ with $\boldsymbol{x}_{i} \in \mathbb{C}^{r+1}$ for $i=1,2$. We shall essentially argue as in Proposition 8.4 of [18] (see also [40], Proposition 8.16). Modulo a translation in $\mathbb{C}^{r+1}$ we can suppose without loss of generality that $\boldsymbol{q}=\mathbf{0}$, that $n\left(\boldsymbol{q}^{\prime}\right) \neq 0$ and that $p_{i}=\left(1: \boldsymbol{x}_{i}: \varphi\left(\boldsymbol{x}_{i}\right): n\left(\boldsymbol{x}_{i}\right)\right) \in X, i=1,2$, are two distinct points such that $q \in\left\langle p_{1}, p_{2}\right\rangle$. We have to show that the equation

$$
q=\lambda p_{1}+\mu p_{2}
$$

has uniquely determined solutions. The above equation splits into the following four:

$$
\lambda+\mu=1, \quad \boldsymbol{x}_{2}=-\frac{\lambda}{\mu} \boldsymbol{x}_{1}, \quad \varphi\left(\boldsymbol{x}_{1}\right)=\frac{\mu}{\lambda} \boldsymbol{q}^{\prime} \quad \text { and } \quad n\left(\boldsymbol{x}_{1}\right)=\frac{\mu^{2}}{\lambda(\mu-\lambda)} z .
$$

Thus relation (5.7) implies

$$
n\left(\boldsymbol{x}_{1}\right) \boldsymbol{x}_{1}=(\ell \circ \varphi)\left(\varphi\left(\boldsymbol{x}_{1}\right)\right)=\frac{\mu^{2}}{\lambda^{2}}(\ell \circ \varphi)\left(\boldsymbol{q}^{\prime}\right)
$$

so that

$$
\boldsymbol{x}_{1}=\frac{\mu-\lambda}{\lambda z}(\ell \circ \varphi)\left(\boldsymbol{q}^{\prime}\right) \quad \text { and } \quad \boldsymbol{x}_{2}=\frac{\lambda-\mu}{\mu z}(\ell \circ \varphi)\left(\boldsymbol{q}^{\prime}\right) .
$$

We deduce that $p_{1}, p_{2} \in X$ are uniquely determined by $q$ as soon as we show that $(\lambda, \mu)$ are uniquely determined by this point. Since $\lambda+\mu=1$, we shall prove that $\lambda \mu$ is uniquely determined.

From relation (5.7), it follows that there exists a cubic form $m$ such that $\varphi \circ \ell \circ$ $\varphi(\boldsymbol{y})=m(\boldsymbol{y}) \boldsymbol{y}$ for every $\boldsymbol{y} \in \mathbb{C}^{r+1}$. This yields $m(\varphi(\boldsymbol{x}))=n(\boldsymbol{x})^{2}$ for every $\boldsymbol{x}$. Applying $m$ to $\varphi\left(\boldsymbol{x}_{1}\right)=\frac{\mu}{\lambda} \boldsymbol{q}^{\prime}$ we deduce $n\left(\boldsymbol{x}_{1}\right)^{2}=\frac{\mu^{3}}{\lambda^{3}} m\left(\boldsymbol{q}^{\prime}\right)$. Combining this with the last relation in (5.8) and remarking that $(\mu-\lambda)^{2}=1-4 \lambda \mu$ (since $\lambda+\mu=1$ ), we finally get

$$
\lambda \mu=\frac{m\left(\boldsymbol{q}^{\prime}\right)}{z^{2}+4 m\left(\boldsymbol{q}^{\prime}\right)},
$$

concluding the proof. 
Remark 5.5. Following a classical approach of Bronowski, see [8], it was proved in [15] that an irreducible variety $X \subset \mathbb{P}^{2 r+3}$ with one apparent double point (OADP variety) projects birationally onto $\mathbb{P}^{r+1}$ from a general tangent space $T=T_{x} X$, see also [16] for generalizations. Letting notation be as in the discussion before Theorem 5.2, then $\pi_{T}(E)=E^{\prime} \subset \mathbb{P}^{r+1}$ is hypersurface of degree $d=\operatorname{deg}\left(E^{\prime}\right) \geq 1$, which is a birational projection of the quadratic Veronese embedding of $\mathbb{P}^{r}$. In particular $1 \leq d \leq 2^{r}$. In Theorem 5.3 of [17] it is proved that for normal OADP varieties having $d=\operatorname{deg}\left(E^{\prime}\right)=1$, not scrolls over a curve, the birational map $\pi_{T}^{-1}: \mathbb{P}^{r+1} \rightarrow X \subset \mathbb{P}^{2 r+3}$ is given by a linear system of cubics hypersurfaces having double points along the base locus of the quadro-quadric Cremona transformation $\pi_{T \mid E}^{-1}: E^{\prime} \rightarrow E$. In particular this class of normal OADP varieties is contained in the class of $X=\bar{X}(r+1,3,3) \subset \mathbb{P}^{2 r+3}$ so that the subsequent classification results of $\bar{X}(r+1,3,3)$ 's of different kind or dimension can be reformulated for the above class of normal varieties, see [17]. Conjecturally every $\bar{X}(r+1,3,3)$ should be projectively equivalent to a $X_{\mathbb{J}} \subset \mathbb{P}^{2 r+3}$, see discussion in Remark 5.13 below. The known examples of twisted cubics over cubic complex Jordan algebras are normal varieties even if we are not aware of a general proof of this fact and neither of the fact that a $\bar{X}(r+1,3,3)$ is a priori normal. If these were true, one would deduce a one to one correspondence between normal OADP varieties with $d=\operatorname{deg}\left(E^{\prime}\right)=1$ and $\bar{X}(r+1,3,3)$ and also probably with twisted cubic over Jordan algebras.

Cremona transformations have been studied since a long time and several classification results have been obtained, especially for quadro-quadric transformations. These classifications can be used to describe all the $\bar{X}(r+1,3,3)$ in low dimension or under suitable hypothesis. We shall begin by recalling some easy results on Cremona transformations of type $(2,2)$ having smooth base loci, see also [20] and [53], Section 4 , for the study of related objects.

Proposition 5.6. Let $\varphi: \mathbb{P}^{r} \rightarrow \mathbb{P}^{r}$ be a Cremona transformation of type $(2,2)$ whose base locus $B \subset \mathbb{P}^{r}$ is smooth. Then one of the following holds:

(1) $r \geq 2, B=Q^{r-2} \amalg p$ with $Q^{r-2}$ a smooth quadric hypersurface and $p \notin$ $\left\langle Q^{\bar{r}-2}\right\rangle$;

(2) $r=5$ and $B$ is projectively equivalent to the Veronese surface $v_{2}\left(\mathbb{P}^{2}\right)$;

(3) $r=8$ and $B$ is projectively equivalent to the Segre variety $\mathbb{P}^{2} \times \mathbb{P}^{2}$;

(4) $r=14$ and $B$ is projectively equivalent to the Grassmann variety $\mathrm{G}_{2}\left(\mathbb{C}^{6}\right)$;

(5) $r=26$ and $B$ is projectively equivalent to the 16-dimensional $E_{6}$ variety.

Proof. Let notation be as in the proof of Corollary 5.3. Let $B_{1}, \ldots, B_{s}$ be the irreducible components of $B$ and let $B_{1}^{\prime}, \ldots, B_{t}^{\prime}$ be the irreducible components of $B^{\prime}$. It is easy to see that the general quadric hypersurface defining $\varphi$ is smooth at every point of $B$. 
The smoothness of $B$ ensures that $B_{j} \cap B_{l}=\emptyset$ for every $j \neq l$ so that $\tilde{\mathbb{P}}^{r}$ is smooth and naturally isomorphic to the successive blow-up of the $B_{i}$ 's in some order. In particular $s=t$ (see also Corollary 5.3).

Let $E_{i}=\pi_{1}^{-1}\left(B_{i}\right)$ and let $E_{i}^{\prime}=\pi_{2}^{-1}\left(B_{i}^{\prime}\right)$. Let $H_{j}=\pi_{j}^{*}(H)$ with $j=1,2$ and with $H \subset \mathbb{P}^{r}$ a hyperplane. We have the following linear equivalence relation of divisors on $\widetilde{\mathbb{P}}^{r}$, see proof of Corollary 5.3:

$$
E_{1}+\cdots+E_{s} \sim 3 H_{2}-2\left(E_{1}^{\prime}+\cdots+E_{s}^{\prime}\right)
$$

and

$$
E_{1}^{\prime}+\cdots+E_{s}^{\prime} \sim 3 H_{1}-2\left(E_{1}+\cdots+E_{s}\right) .
$$

Thus the scheme-theoretic images $\pi_{2}\left(E_{1}+\cdots+E_{s}\right)$ and $\pi_{1}\left(E_{1}^{\prime}+\cdots+E_{s}^{\prime}\right)$ have degree 3, yielding $s \leq 3$.

Suppose $s=3$. Then $\operatorname{deg}\left(\pi_{2}\left(E_{i}\right)\right)=\operatorname{deg}\left(\pi_{1}\left(E_{i}^{\prime}\right)\right)=1$ for every $i=1,2,3$ so that $B_{i}$ and $B_{i}^{\prime}$ are a linearly embedded $\mathbb{P}^{r-2}$ since the intersection of two distinct hyperplanes $\pi_{1}\left(E_{i}^{\prime}\right)$, respectively $\pi_{2}\left(E_{i}\right)$, is contained in the base locus. The smoothness of a general quadric defining $\varphi$ along each $B_{i}=\mathbb{P}^{r-2} \subset \mathbb{P}^{r}$ implies $r-2 \leq \frac{r-1}{2}$, that is $r \leq 3$. Thus necessarily $r=2$ since $h^{0}\left(\mathcal{I}_{B}(2)\right)=r+1$ and we are in case (1).

If $s=2$, we can suppose $\operatorname{deg}\left(\pi_{1}\left(E_{1}^{\prime}\right)\right)=2$ and $\operatorname{deg}\left(\pi_{1}\left(E_{2}^{\prime}\right)\right)=1$. Thus the quadric $\pi_{1}\left(E_{1}^{\prime}\right) \cap \pi_{1}\left(E_{2}^{\prime}\right)$ is an irreducible component, let us say $B_{1}$, of $B$. The birationality of $\varphi$ implies $h^{0}\left(\mathcal{J}_{B}(2)\right)=r+1$. Since $h^{0}\left(\mathcal{J}_{B}(2)\right) \leq h^{0}\left(\mathcal{J}_{B_{1}}(2)\right)=r+2$, we see that $B_{2}$ consists of only one point and we are in case (1).

If $s=1$, the above diagram (5.4) shows that for general $q \in \pi_{1}\left(E_{1}^{\prime}\right) \backslash B$ there exists a linear space $\mathbb{P}_{q}^{r-1-\operatorname{dim}\left(B^{\prime}\right)}$ passing through $q$ and cutting $X$ along a quadric hypersurface of dimension $r-2-\operatorname{dim}(B)$. If $\varphi(q)=q^{\prime}$, then naturally $\mathbb{P}^{r-1-\operatorname{dim}\left(B^{\prime}\right)}=\mathbb{P}\left(\left(N_{B^{\prime} / \mathbb{P}^{r}}\right)_{x}^{*}\right)$. This immediately implies that $\pi_{1}\left(E_{1}^{\prime}\right)$ is the variety of secant lines to $B$ and that $B \subset \mathbb{P}^{r}$ is a $Q E L$-manifold of type $\delta(B)=\frac{1}{2} \operatorname{dim}(B)$, see [53], Proposition 4.2. Indeed, $r-2-\operatorname{dim}(B)=\delta(B)=2 \operatorname{dim}(B)+1-\operatorname{dim}\left(\pi_{1}\left(E_{1}^{\prime}\right)\right)$ yields $\operatorname{dim}(B)=\frac{2}{3}(r-2)$, see also the computations in [20]. Thus $B \subset \mathbb{P}^{r}$ is a $Q E L$-manifold and also a Severi variety in the sense of Zak. The classification of Severi varieties due to Zak, see [68] and also [53], Corollary 3.2, assures that we are in one of the cases (3)-(6).

The classification of arbitrary $\bar{X}(r+1,3,3) \subset \mathbb{P}^{2 r+3}$ is difficult due to the existence of a lot of singular examples. By Theorem 5.2 and Corollary 5.3 this classification is closely related to that of all Cremona involutions of type $(2,2)$ on $\mathbb{P}^{r}$ and also to the classification of arbitrary complex cubic Jordan algebras of dimension $r+1$. On the contrary for smooth $\bar{X}(r+1,3,3)$ 's a complete classification is possible due to Proposition 5.6 and Theorem 5.2.

Theorem 5.7. Let $X=\bar{X}(r+1,3,3) \subset \mathbb{P}^{2 r+3}$ be smooth. Then one of the following holds, modulo projective equivalence: 
(i) $X$ is either $S_{1 \ldots 122}$ or $S_{1 \ldots 113}$;

(ii) $X$ is the Segre embedding $\mathbb{P}^{1} \times Q^{r} \subset \mathbb{P}^{2 r+3}$ with $Q^{r}$ a smooth hyperquadric;

(iii) $r=5$ and $X$ is the Lagrangian Grassmannian $\mathrm{LG}_{3}\left(\mathbb{C}^{6}\right) \subset \mathbb{P}^{13}$;

(iv) $r=8$ and $X$ is the Grassmannian $\mathrm{G}_{3}\left(\mathbb{C}^{6}\right) \subset \mathbb{P}^{19}$;

(v) $r=14$ and $X$ is the orthogonal Grassmannian $\mathrm{OG}_{6}\left(\mathbb{C}^{12}\right) \subset \mathbb{P}^{31}$;

(vi) $r=26$ and $X$ is the $E_{7}$-variety in $\mathbb{P}^{55}$.

Proof. If the associated Cremona transformation is equivalent to a projective transformation we are in case (i) by Theorem 5.2. Otherwise, by Theorem 5.2, the associated Cremona transformation $\psi_{x}$ is of type $(2,2)$ with smooth base locus. Let $\phi: \mathbb{P}^{r+1} \rightarrow X \subset \mathbb{P}^{2 r+3}$ be the birational representation of $X$ given by the linear system of cubic hypersurfaces having double points along $B_{x}^{\prime}$. Then $B_{x}^{\prime}$ is projectively equivalent to a variety as in cases (1)-(5) of Proposition 5.6 so that $X$ is as in cases (ii)-(vi) by a well-known description of the corresponding varieties, see for example [38], [39], [46].

Now we apply the classification of quadro-quadric Cremona transformations in low dimension to deduce the corresponding classification for varieties 3 -covered by twisted cubics. For instance, since every birational map $\varphi: \mathbb{P}^{1} \rightarrow \mathbb{P}^{1}$ is equivalent to a projective transformation, one immediately deduces that a surface $\bar{X}(2,3,3) \subset \mathbb{P}^{5}$ is necessarily of Castelnuovo type, namely it is one of the scrolls $S_{13}$ or $S_{22}$. Then, by projecting from $m-3$ general points, one gets the following result:

Corollary 5.8. Let $X=\bar{X}(2, m, m) \subset \mathbb{P}^{m+2}, m \geq 3$. Then $X$ is projectively equivalent to a smooth rational normal scroll of degree $m+1$.

The classification of birational maps of degree 2 on $\mathbb{P}^{2}$ is classical, easy and well known. From this we shall immediately deduce the classification of arbitrary $\bar{X}(3,3,3) \subset \mathbb{P}^{7}$.

Corollary 5.9. Let $X=\bar{X}(3,3,3) \subset \mathbb{P}^{7}$. Then $X$ is projectively equivalent to

(1) a smooth rational normal scroll of degree 5, that is $S_{113}$ or $S_{122}$; or

(2) the variety $\mathbb{P}^{1} \times Q^{2} \subset \mathbb{P}^{7}$ where $Q^{2} \subset \mathbb{P}^{3}$ is an irreducible quadric; or

(3) the normal del Pezzo 3-fold obtained as the image of $\mathbb{P}^{3}$ by the linear system of cubic surfaces having three infinitely near double points or equivalently described as the twisted cubic over the Jordan algebra $A_{3}$ of Theorem 4.12 and admitting the parametrization (4.8). 
Proof. If $\psi_{x}: \mathbb{P}^{2} \rightarrow \mathbb{P}^{2}$ is equivalent to a projective transformation, then we are in case (1) by Proposition 5.2. Otherwise $\psi_{x}: \mathbb{P}^{2} \rightarrow \mathbb{P}^{2}$ is a Cremona transformation of type $(2,2)$. If $\psi_{x}$ is the ordinary quadratic transformation, then the cubic surfaces defining $\phi: \mathbb{P}^{3} \rightarrow X \subset \mathbb{P}^{7}$ have three double distinct points at the indeterminacy points of $\phi_{x}^{-1}$ so that this linear system coincides with the complete linear system of cubic surfaces having these three double points. In this case $X \subset \mathbb{P}^{7}$ is projectively equivalent to the Segre embedding of $\mathbb{P}^{1} \times Q^{2}$ with $Q^{2}$ a smooth quadric surface, see also Theorem 5.7.

If $\psi_{x}$ has two infinitely near base points and another base point, reasoning as above we have that $X \subset \mathbb{P}^{7}$ is projectively equivalent to the Segre embedding of $\mathbb{P}^{1} \times S_{02}$.

If $\psi_{x}$ has three infinitely near base points, then $\phi$ is given by a linear system of cubic surfaces having three infinitely near double points and we are in case (3).

Using the last result, we can now classify irreducible 3-folds $X=\bar{X}(3, m, m) \subset$ $\mathbb{P}^{m+4}$ for every $m \geq 4$. If $m \geq 4$, by projecting from $m-3$ general points we get an irreducible 3-fold $X_{m-3} \subset \mathbb{P}^{7}$ which is 3-covered by twisted cubics. If $X_{m-3}$ is a smooth rational normal scroll of degree 5 , then $X \subset \mathbb{P}^{m+4}$ is a smooth rational normal scroll of degree $m+2$. If $X_{m-3} \subset \mathbb{P}^{7}$ is a 3-fold as in Corollary 5.9, then $X \subset \mathbb{P}^{m+4}$ would be a normal del Pezzo 3-fold of degree $6+m-3=m+3 \geq 7$, which is linearly normal. Moreover since $S X_{m-3}=\mathbb{P}^{7}$, we deduce $\operatorname{dim}(S X)=7$. The normal del Pezzo 3-folds $X \subset \mathbb{P}^{m+4}$ of degree $m+3, m \geq 4$, are smooth and with $\operatorname{dim}(S X)=6$, see [25]. In conclusion we have proved the following result:

Corollary 5.10. Let $X=\bar{X}(3, m, m) \subset \mathbb{P}^{m+4}$ with $m \geq 4$. Then $X$ is a smooth rational normal scroll of degree $m+2$.

Also the classification of quadratic Cremona transformation on $\mathbb{P}^{3}$ is known. By comparing Tableau 1 in [47] and Table 2 above one obtains the following result.

Theorem 5.11. Let $\psi: \mathbb{P}^{3} \rightarrow \mathbb{P}^{3}$ be a Cremona transformation of bidegree $(2,2)$, not equivalent to a projective transformation. Then up to composition to the right and to the left by linear automorphisms, $\psi$ can be assumed to be one of the seven quadratic involutions $x \mapsto x^{\#}$ defining the adjoint of one of the seven cubic Jordan algebras on $\mathbb{C}^{4}$ described in Section 4.4.2 (see Table 2 for explicit formulae).

From Theorem 5.11, it follows immediately the

Corollary 5.12. Let $X=\bar{X}(4,3,3) \subset \mathbb{P}^{9}$. Then $X$ is projectively equivalent to one of the following varieties:

(1) a smooth rational normal scroll of degree 6 , that is $S_{1113}$ or $S_{1122}$; or

(2) a cubic curve over one of the seven Jordan algebras in Table 2. 
Remark 5.13. The varieties appearing in Theorem 5.7 are also particular examples of smooth Legendrian varieties, see [41] for definitions, some related results and references. They are also interesting examples of smooth varieties with one apparent double point defined before by Corollary 5.4, see also [46] and [15].

The class of Cremona transformations $\psi: \mathbb{P}^{r} \rightarrow \mathbb{P}^{r}$ of type $(2,2)$ arising from two different birational projections from one point of an irreducible quadric hypersurface $Q^{r}$ will be called elementary quadratic transformations, that is if $\pi_{1}: Q^{r} \rightarrow \mathbb{P}^{r}$ and if $\pi_{2}: Q^{r} \rightarrow \mathbb{P}^{r}$ are the two projections we have $\psi=\pi_{2} \circ \pi_{1}^{-1}$. The well-known classification of plane quadratic Cremona transformations and the results of Theorem 5.11 say that for $r \leq 3$ every Cremona transformation of type $(2,2)$ is an elementary quadratic transformation. These examples yield varieties $\bar{X}(r+1,3,3) \subset \mathbb{P}^{2 r+3}$, not rational normal scrolls, which are either the Segre embedding of $\mathbb{P}^{1} \times Q^{r}$ or projective degenerations of them when some base point becomes infinitely near.

There is an interesting approach to Jordan algebras developed by T. A. Springer [61], Section 1.27, and based on $j$-structures and indirectly also on the so-called Hua's identities, see [44]. These results and our geometrical treatment yield the following consequence, probably well known to the experts: two twisted cubic curves over Jordan algebras $X_{\mathbb{J}_{i}} \subset \mathbb{P}^{2 r+3}, i=1,2$, are projectively equivalent if and only if $\mathbb{J}_{1}$ and $\mathbb{J}_{2}$ are isomorphic Jordan algebras.

In particular in Theorem 5.7, Corollary 5.9 and Corollary 5.12 we obtained geometrical proofs of the classification of all cubic Jordan algebras $\mathbb{J}$ such that the associated twisted cubic $X_{\mathbb{J}} \subset \mathbb{P}^{2 r+3}$ is respectively smooth, of dimension 3 , of dimension 4.

Based on the results of Theorem 5.2, of the construction in the proof of Corollary 5.3, of Theorem 5.7, of Corollary 5.9 and of Corollary 5.12, one could ask the following question:

Is a $\bar{X}(r+1,3,3) \subset \mathbb{P}^{2 r+3}$ not of Castelnuovo type projectively equivalent to a twisted cubic $X_{\mathbb{J}} \subset \mathbb{P}^{2 r+3}$ for some cubic Jordan algebra $\mathbb{J}$ of dimension $r+1$ ?

We conjecture that the answer to the previous question is affirmative. In other terms, we conjecture that the following a priori unrelated mathematical objects in fact coincide:

- varieties $\bar{X}(r+1,3,3) \subset \mathbb{P}^{2 r+3}$, up to projective equivalence;

- rank 3 Jordan algebras of dimension $r+1$, up to isomorphism;

- quadro-quadratic Cremona transformations of $\mathbb{P}^{r}$, up to linear equivalence.

In [9], see also [60] and [66], a classification of quadro-quadric Cremona transformations in $\mathbb{P}^{4}$ is obtained. This immediately yields also the classification of $\bar{X}(5,3,3) \subset \mathbb{P}^{11}$ and provides an affirmative answer to the above conjecture also for $r=4$. We refrain from listing this classification and we will come back in a future 
paper, [50], on the beautiful relations between the above apparently unrelated objects, trying to develop further the classification results and the connections among these areas.

\section{References}

[1] L. Bădescu, M. C. Beltrametti, and P. Ionescu, Almost-lines and quasi-lines on projective manifolds. In Complex analysis and algebraic geometry, in memory of Michael Schneider, de Gruyter, Berlin 2000, 1-27. Zbl 1078.14010 MR 1760869

[2] J. Baez, The octonions. Bull. Amer. Math. Soc. 39 (2002), 145-205. Zbl 1026.17001 MR 1886087

[3] D. Barlet, Le faisceau $\omega_{X}^{\bullet}$ sur un espace analytique $X$ de dimension pure. In Fonctions de plusieurs variables complexes. III, Lecture Notes in Math. 670, Springer-Verlag, Berlin 1978, 187-204. Zbl 0398.32009 MR 0521919

[4] G. Bol, Flächengewebe im dreidimensionalen Raum. Abh. Math. Semin. Univ. Hamburg 10 (1934), 119-133. Zbl 0009.18105

[5] G. Bol, Über eine bemerkenswerte Familie zweidimensionaler Flächen. Abh. Math. Semin. Univ. Hamburg 10 (1934), 309-316. Zbl 0009.18202 MR 3069634

[6] E. Bompiani, Proprietà differenziali caratteristiche di enti algebrici. Rom. Acc. L. Mem. 26 (1921), 452-474. JFM 48.0851.04

[7] E. Bompiani, Über Flächen, die ein dreiparametriges System rationaler Normalkurven enthalten. Abh. Math. Semin. Univ. Hamburg 10 (1934), 305-308. Zbl 0009.18201 MR 3069633

[8] J. Bronowski, The sum of powers as canonical expressions. Proc. Cambridge Philos. Soc. 29 (1932), 69-82. Zbl 0006.09905

[9] A. Bruno and A. Verra, The quadro-quadric Cremona transformations of $P^{4}$ and $P^{5}$. Mem. Accad. Sci. Torino, Cl. Sci. Fis. Mat. Nat. (5) 35 (2011), 3-21. Zbl 06151766

[10] F. Campana, Coréduction algébrique d'un espace analytique faiblement kählérien compact. Invent. Math. 63 (1981), 187-223. Zbl 0436.32024 MR 0610537

[11] G. Castelnuovo, Massima dimensione dei sistemi lineari di curve piane di dato genere. Annali di Mat. 18 (1890), 119-128. JFM 22.0627.01

[12] G. Castelnuovo, Ricerche generali sopra i sistemi lineari di curve piane. Mem. R. Accad. Sci. Torino Cl. Sci. Fis. Mat. Nat. 42 (1890-1891), 137-188. JFM 23.0653.01

[13] S. S. Chern, P. A. Griffiths, An inequality for the rank of a web and webs of maximum rank. Ann. Scuola Norm. Sup. Pisa 5 (1978), 539-557. Zbl 0402.57001 MR 0507000

[14] C. Ciliberto, On a property of Castelnuovo varieties. Trans. Amer. Math. Soc. 303 (1987), 201-210. Zbl 0657.14022 MR 0896017

[15] C. Ciliberto, M. Mella, and F. Russo, Varieties with one apparent double point. J. Algebraic Geom. 13 (2004), 475-512. Zbl 1077.14076 MR 2047678 
[16] C. Ciliberto and F. Russo, Varieties with minimal secant degree and linear systems of maximal dimension on surfaces. Adv. Math. 200 (2006), 1-50. Zbl 1086.14043 MR 2199628

[17] C. Ciliberto and F. Russo, On the classification of OADP varieties. Sci. China Math. 54 (2011), no. 8, 1561-1575. Zbl 1246.14068 MR 2824959

[18] J.-L. Clerc, Special prehomogeneous vector spaces associated to $F_{4}, E_{6}, E_{7}, E_{8}$ and simple Jordan algebras of rank 3. J. Algebra 264 (2003), 98-128. Zbl 1034.17006 MR 1980688

[19] G. Darboux, Sur le contact des courbes et des surfaces. Bull. Soc. Math. France 4 (1880), 348-384. JFM 12.0590.01

[20] L. Ein and N. Shepherd-Barron, Some special Cremona transformations. Amer. J. Math. 111 (1989), 783-800. Zbl 0708.14009 MR 1020829

[21] D. Eisenbud and J. Harris, On varieties of minimal degree (a centennial account). Proc. Sympos. Pure Math. 46 (1987), 3-13. Zbl 0646.14036 MR 0927946

[22] F. Enriques, Sulla massima dimensione dei sistemi lineari di dato genere appartenenti a una superficie algebrica. Atti Reale Acc. Scienze Torino 29 (1894), 275-296. Zbl 25.1217.01

[23] A. Fialowski, M. Penkava, and M. Phillipson, Deformations of complex 3-dimensional associative algebras. J. Gen. Lie Theory Appl. 5 (2011), Art. ID G110102, 22 pp. Zbl 1223.14012 MR 2846731

[24] J. Faraut and A. Korányi, Analysis on symmetric cones. Oxford Math. Monogr., Oxford University Press, New York 1994. Zbl 0841.43002 MR 1446489

[25] T. Fujita, Projective varieties of $\Delta$-genus one. In Algebraic and topological theories, Kinokuniya, Tokyo 1986, 149-175. Zbl 0800.14020 MR 1102257

[26] P. Gabriel, Finite representation type is open. In Proceedings of the International Conference on Representations of Algebras, Paper No. 10, Carleton Math. Lecture Notes 9, Carleton University, Ottawa, Ont., 1974. Zbl 0313.16034 MR 0376769

[27] P. A. Griffiths, Variations on a theorem of Abel. Invent. Math. 35 (1976), 321-390. Zbl 0339.14003 MR 0435074

[28] P. A. Griffiths and J. Harris, Principles of algebraic geometry. Pure and Applied Mathematics, Wiley-Interscience, New York, 1978. Zbl 0408.14001 MR 0507725

[29] P. A. Griffiths and J. Harris, Algebraic geometry and local differential geometry. Ann. Sci. Ecole Norm. Sup. 12 (1979), 355-432. Zbl 0426.14019 MR 0559347

[30] J. Harris, A bound on the geometric genus of projective varieties. Ann. Scuola Norm. Sup. Pisa 8 (1981), 35-68. Zbl 0467.14005 MR 0616900

[31] G. Henkin and M. Passare, Abelian differentials on singular varieties and variations on a theorem of Lie-Griffiths. Invent. Math. 135 (1999), 297-328. Zbl 0932.32012 MR 1666771

[32] P. Ionescu, Birational geometry of rationally connected manifolds via quasi-lines. In Projective varieties with unexpected properties (Siena, 2004), de Gruyter, Berlin 2005, 317-335. Zbl 1109.14037 MR 2202261

[33] P. Ionescu and F. Russo, Conic-connected manifolds. J. Reine Angew. Math. 644 (2010), 145-157. Zbl 1200.14078 MR 2671777 
[34] N. Jacobson, Structure and representations of Jordan algebras. Amer. Math. Soc. Colloquium Publications XXXIX, Amer. Math. Soc., Providence, RI, 1968. Zbl 0218.17010 MR 0251099

[35] J. Kollár, Rational curves on algebraic varieties. Ergeb. Math. Grenzgeb. 32, SpringerVerlag, Berlin 1996. Zbl 0877.14012 MR 1440180

[36] J. Kollár, Y. Miyaoka, and S. Mori, Rationally connected varieties. J. Algebraic Geom. 1 (1992), 429-448. Zbl 0780.14026 MR 1158625

[37] E. Kunz and R. Waldi, Regular differential forms. Contemp. Math. 79, Amer. Math. Soc., Providence, RI, 1988. Zbl 0658.13019 MR 0971502

[38] J. M. Landsberg and L. Manivel, Construction and classification of complex simple Lie algebras via projective geometry. Selecta Math. (N.S.) 8 (2002), 137-159. Zbl 1073.14551 MR 1890196

[39] J. M. Landsberg and L. Manivel, The projective geometry of Freudenthal's magic square. J. Algebra 239 (2001), 477-512. Zbl 1064.14053 MR 1832903

[40] J. M. Landsberg and L. Manivel, The sextonions and $E_{7 \frac{1}{2}}$. Adv. Math. 201 (2006), 143-179. Zbl 1133.17007 MR 2204753

[41] J. M. Landsberg and L. Manivel, Legendrian varieties. Asian J. Math. 11 (2007), 341-360. Zbl 1136.14024 MR 2372722

[42] E. P. Lane, A treatise on projective differential geometry. University of Chicago Press, Chicago, Ill., 1942. Zbl 0063.03443 MR 0007286

[43] J. Lipman, Dualizing sheaves, differentials and residues on algebraic varieties. Astérisque 117 (1984). Zbl 0562.14003 MR 0759943

[44] K. Mc Crimmon, A taste of Jordan algebras. Universitext, Springer-Verlag, New York 2004. Zbl 1044.17001 MR 2014924

[45] S. Mori, Projective manifolds with ample tangent bundles. Ann. of Math. 110 (1979), 593-606. Zbl 0423.14006 MR 0554387

[46] S. Mukai, Simple Lie algebra and Legendre variety. Preprint 1998; available at http://www.kurims.kyoto-u.ac.jp/ mukai/paper/warwick15.pdf

[47] I. Pan, F. Ronga, and T. Vust, Transformations birationelles quadratiques de l'espace projectif complexe à trois dimensions. Ann. Inst. Fourier Grenoble 51 (2001), 1153-1187. Zbl 0987.14009 MR 1860661

[48] V. Paterno, Special rationally connected manifolds. Ph.D. Thesis, Trento, December 2009.

[49] J. V. Pereira and L. Pirio, An invitation to web geometry: from Abel's Addition Theorem to the algebraization of codimension one webs. Publicações Matematicas, IMPA, 2009. Zbl 1184.53002 MR 2536234

[50] L. Pirio and F. Russo, Extremal varieties 3-rationally connected by cubics, quadroquadric Cremona transformations and rank 3 Jordan algebras. Preprint 2011, arXiv:1109.3573 [math.AG].

[51] L. Pirio and J.-M. Trépreau, Sur l'algébrisation des tissus de rang maximal. Preprint 2013, arXiv:1302.3142 [math.AG]. 
[52] L. Pirio and J.-M. Trépreau, Sur les variétés $X \subset \mathbb{P}^{N}$ telles que par $n$ points passe une courbe de $X$ de degré donné. Bull. Soc. Math. France 141 (2013), no. 1, 131-196. Zbl 06161945 MR 3031675

[53] F. Russo, Varieties with quadratic entry locus, I. Math. Ann. 344 (2009), 597-617. Zbl 1170.14040 MR 2501303

[54] F. Russo, Geometry of special varieties. Available at http://www.dmi.unict.it/ frusso/DMI/Note_di_Corso_files/GeometrySpecialVarieties.pdf

[55] G. Scorza, Sulla determinazione delle varietà a tre dimensioni di $S_{r}(r \geq 7)$ i cui $S_{3}$ tangenti si tagliano a due a due. Rend. Circ. Mat. Palermo 25 (1908), 193-204. JFM 39.0717.01

[56] G. Scorza, Sulle varietà a quattro dimensioni di $S_{r}(r \geqq 9)$ i cui $S_{4}$ tangenti si tagliano a due a due. Rend. Circ. Mat. Palermo 27 (1909), 148-178. JFM 40.0712 .01

[57] G. Scorza, Le algebre del 3. ${ }^{\circ}$ ordine. Atti Accad. Sci. Fis. Mat. Napoli II. Ser. 20, 13 (1935), 1-14. JFM 61.1035.02

[58] G. Scorza, Le algebre del 4. ${ }^{\circ}$ ordine. Atti Accad. Sci. Fis. Mat. Napoli, II. Ser. 2014 (1935), 1-83. JFM 61.1035.02

[59] C. Segre, Le superficie degli iperspazi con una doppia infinità di curve piane o spaziali. Atti Acc. Torino 56 (1921), 143-517; 57 (1922), 575-585. JFM 61.1036.01

[60] J. G. Semple, Cremona transformations of space of four dimensions by means of quadrics, and the reverse transformations. Philos. Trans. Roy. Soc. London Ser. A 228 (1929), 331-376. JFM 55.0991.02

[61] T. Springer, Jordan algebras and algebraic groups. Classics Math., Springer-Verlag, Berlin 1998. Zbl 1024.17018 MR 1490836

[62] E. Study, Über Systeme complexer Zahlen und ihre Anwendung in der Theorie der Transformationsgruppen. Monatsh. Math. 1 (1890), 283-355. JFM 22.0387.02 MR 1546158

[63] J.-M. Trépreau, Algébrisation des tissus de codimension 1. La généralisation d'un théorème de Bol. In Inspired by S. S. Chern, Nankai Tracts Math. 11, World Scientific Publishing, Hackensack, NJ, 2006, 399-433. Zbl 1136.53010 MR 2313344

[64] J.-M. Trépreau, Une nouvelle caractérisation des variétés de Veronese. Preprint 2010, arXiv:1012.1008 [math.AG].

[65] G. Veronese, Behandlung der projectivischen Verhältnisse der Räume von verschiedenen Dimensionen durch das Princip des Projicirens und Schneidens. Math Ann. 19 (1882), 161-234. JFM 13.0485.01 MR 1510125

[66] A. Verra, Lectures on Cremona transformations. Torino, September 19-24, 2005; preprint 2005, available at http://www.mat.uniroma3.it/users/verra/lectures.html

[67] B. Westbury, Sextonions and the magic square. J. London Math. Soc. 73 (2006), 455-474. Zbl 1154.17304 MR 2225497

[68] F. L. Zak, Tangents and secants of algebraic varieties, Transl. Math. Monogr. 127, Amer. Math. Soc., Providence, RI, 1993. Zbl 0795.14018 MR 1234494 
Vol. 88 (2013)

Luc Pirio, IRMAR, UMR 6625 du CNRS, Université Rennes1, Campus de beaulieu, 35000 Rennes, France

E-mail: luc.pirio@univ-rennes1.fr

Francesco Russo, Dipartimento di Matematica e Informatica Università degli Studi di Catania 95125 Catania, Italy

E-mail: frusso@dmi.unict.it 